\title{
Privacy with Imperfect Randomness
}

\author{
Yevgeniy Dodis $^{a}$ Yanqing Yao ${ }^{b, a}$ \\ ${ }^{a}$ Department of Computer Science, New York University, New York 10012, USA \\ ${ }^{b}$ State Key Laboratory of Software Development Environment, Beihang University, Beijing 100191, China \\ dodis@cs.nyu.edu, yaoyanqing1984@gmail.com
}

\begin{abstract}
We revisit the impossibility of a variety of cryptographic tasks including privacy and differential privacy with imperfect randomness. For traditional notions of privacy, such as security of encryption, commitment or secret sharing schemes, dramatic impossibility results are known [MP90,DOPS04] for several concrete sources $\mathcal{R}$, including a (seemingly) very "nice and friendly" Santha-Vazirani (SV) source. Moreover, Bosley and Dodis [BD07] gave strong evidence that many traditional privacy tasks
\end{abstract} (e.g., encryption) inherently require an "extractable" source of randomness.

The common interpretation of these negative results is that privacy is impossible even with "very structured" imperfect sources. Somewhat surprisingly, Dodis et al. [DLMV12] put a slight dent in this belief, by showing that non-trivial differential privacy is possible with the SV sources. This suggested a qualitative gap between traditional and differential privacy, and left open the question if differential privacy is possible with more realistic (i.e., less structured) sources than the SV sources.

Motivated by solving this question, we introduce a new, modular framework for showing strong impossibility results for (either traditional or differential) privacy under a general imperfect source $\mathcal{R}$. In particular, we introduce natural and easy-to-understand concepts of expressiveness and separability of a given imperfect source $\mathcal{R}$, and show the following results:

- Low levels of expressiveness generically imply strong impossibility results for both traditional and differential privacy.

- Separability implies expressiveness; NON-separability is equivalent to "weak bit extraction".

- While the separability of some concrete (e.g., SV) sources $\mathcal{R}$ was implicitly known, we show new separability results for several important sources, including general "block sources".

As direct corollaries of these general results, we get the following corollaries:

- Existing, but quantitatively improved, impossibility results for traditional privacy, but under a wider variety of sources $\mathcal{R}$.

- First impossibility results for differential privacy. Although, unsurprisingly, these results (barely) miss the highly structured SV sources, they come back extremely quickly once the source becomes slightly more realistic (e.g., a realistic "block source").

- Any imperfect source allowing (either traditional or differential) privacy admits a certain type of deterministic bit extraction. (This result is incomparable to the result of [BD07].)

Overall, we believe our results provide an intuitive, modular and unified picture elucidating the (im)possibility of privacy with general imperfect sources.

Keywords: imperfect randomness, entropy sources, Santha-Vazirani sources, block sources, BiasControl Limited sources, randomness extraction, privacy, differential privacy 


\section{Introduction}

Traditional cryptographic tasks take for granted the availability of perfect random sources, i.e., sources that output unbiased and independent random bits. However, in many situations it seems unrealistic to expect a source to be perfectly random, and one must deal with various imperfect sources of randomness. Some well known examples of such imperfect random sources are physical sources [BST03,BH05], biometric data $\left[\mathrm{BDK}^{+} 05, \mathrm{DORS08}\right]$, secrets with partial leakage, and group elements from Diffie-Hellman key exchange [GKR04,Kra10].

IMPERfECT Sources. To abstract this concept, several formal models of realistic imperfect sources have been described (e.g., [vN51,CFG ${ }^{+}$85,Blu86,SV86,CG88,LLS89,Zuc96,ACRT99,Dod01]). Roughly, they can be divided into extractable and non-extractable. Extractable sources (e.g., [vN51,CFG ${ }^{+} 85$, Blu86,LLS89]) allow for deterministic extraction of nearly perfect randomness. And, while the question of optimizing the extraction rate and efficiency has been very interesting, from the qualitative perspective such sources are good for any application where perfect randomness is sufficient. Unfortunately, it was quickly realized that many realistic sources are non-extractable [SV86,CG88,Dod01]. The simplest example of such a source is the Santha-Vazirani (SV) source [SV86], which produces an infinite sequence of (possibly correlated) bits $r_{1}, r_{2}, \ldots$, with the property that $\operatorname{Pr}\left[r_{i}=0 \mid r_{1} \ldots r_{i-1}\right] \in\left[\frac{1}{2}(1-\gamma), \frac{1}{2}(1+\gamma)\right]$, for any setting of the prior bits $r_{1}, r_{2}, \ldots, r_{i-1}$. However, despite the fact that each bit has almost one bit of fresh entropy, Santha and Vazirani [SV86] showed that there exists no deterministic extractor Enc : $\{0,1\}^{n} \rightarrow\{0,1\}$ capable of extracting even a single bit of bias strictly less than $\gamma$ from the $\gamma$-SV source, irrespective of how many SV bits $r_{1}, r_{2}, \ldots, r_{n}$ it is willing to wait for.

Despite this pessimistic result, ruling out the "black-box compiler" from perfect to imperfect (e.g., SV) randomness for all applications, one may still hope that specific "non-extractable" sources, such as SVsources, might be sufficient for concrete applications, such as simulating probabilistic algorithms or cryptography. Indeed, a series of results [VV85,SV86,CG88,Zuc96,ACRT99] showed that very "weak" sources (including SV-sources and even much more realistic "weak" and "block" sources) are sufficient for simulating probabilistic polynomial-time algorithms; namely, for problems which do not inherently need randomness, but which could potentially be sped up using randomization. Moreover, even in the area of cryptography where randomness is essential (e.g., for key generation) - it turns out that many "non-extractable" sources (again, including SV sources and more) are sufficient for authentication applications, such as the designs of MACs [MW97,DKRS06] and even signature schemes [DOPS04,ACM ${ }^{+}$14] (under appropriate hardness assumptions). Intuitively, the reason for the latter "success story" is that authentication applications only require that it is hard for the attacker to completely guess (i.e., "forge") some long string, so having (min)entropy in our source should be sufficient to achieve this goal.

Negative Results for Privacy with Imperfect Randomness. In contrast, the situation appears to be much less bright when dealing with privacy applications, such as encryption, commitment, zero-knowledge, and a few others. First, McInnes and Pinkas [MP90] showed that unconditionally secure symmetric encryption cannot be based on SV sources, even if one is restricted to encrypting a single bit. This result was subsequently strengthened by Dodis et al. [DOPS04], who showed that SV sources are not sufficient for building even computationally secure encryption (again, even of a single bit), and, in fact, essentially any other cryptographic task involving "privacy" (e.g., commitment, zero-knowledge, secret sharing and others). This was again strengthened by Austrin et al. $\left[\mathrm{ACM}^{+} 14\right]$, who showed that the negative results still hold even if the SV source is efficiently samplable. Finally, Bosley and Dodis [BD07] showed an even more negative result: if a source of randomness $\mathcal{R}$ is "good enough" to generate a secret key capable of encrypting $k$ bits, then one can deterministically extract nearly $k$ almost uniform bits from $\mathcal{R}$, suggesting that traditional privacy requires an "extractable" source of randomness. ${ }^{1}$

What about Differential Privacy? While the above series of negative results seem to strongly point in the direction that privacy inherently requires extractable randomness, a recent work of Dodis et al. [DLMV12] put a slight dent into this consensus, by showing that SV sources are provably sufficient for achieving a more recent notion of privacy, called differential privacy [DMNS06]. Intuitively, a differentially private mechanism

\footnotetext{
${ }^{1}$ On the positive side, [DS02] and [BD07] showed that extractable sources are not strictly necessary for encrypting a "very small" number of bits. Still, for natural "non-extractable" sources, such as SV sources, it is known that encrypting even a single bit is impossible [SV86,DOPS04, $\left.\mathrm{ACM}^{+} 14\right]$.
} 
$M(D, \mathbf{r})$ uses its randomness $\mathbf{r}$ to add some "noise" to the true answer $q(D)$, where $D$ is some sensitive database of users, and $q$ is some useful aggregate information (query) about the users of $D$. This noise is added in a way as to satisfy the following two conflicting properties (see Definitions 6 and 7 for formalism):

(a) $\varepsilon$-differential privacy $(\varepsilon$-DP): up to "advantage" $\varepsilon$, the returned value $z=M(D, \mathbf{r})$ does not tell any information about the value $D(i)$ of any individual user $i$, which was not already known to the attacker before $z$ was returned; and

(b) $\rho$-utility: on average (over $\mathbf{r}$ ), $|z-q(D)|$ is upper bounded by $\rho$, meaning that perturbed answer is not too far from the true answer.

Since we will be mainly talking about negative results, for the rest of this work we will restrict our attention to the simplest concrete example of differential privacy, where a "record" $D(i)$ is a single bit, and $q$ is the Hamming weight $w t(D)$ of the corresponding bit-vector $D$ (i.e., $w t(D)=\sum D(i)$ ). In this case, a very simple $\varepsilon$-DP mechanism [DMNS06] $M(D, \mathbf{r})$ would simply return $w t(D)+e(\mathbf{r})$ (possibly truncated to always be between 0 and $|D|)$, where $e(\mathbf{r})$ is an appropriate noise ${ }^{2}$ with $\rho=\mathbb{E}[|q(\mathbf{r})|] \approx 1 / \varepsilon$. Intuitively, this setting ensures that when the value $D(i)$ changes from 0 to 1 , the answer distribution $M(D, \mathbf{r})$ does not "change" by more than $\varepsilon$.

Coming back to Dodis et al. [DLMV12], the authors show that although no "additive noise" mechanism of the form $M(D, \mathbf{r})=w t(D)+e(\mathbf{r})$ can simultaneously withstand all $\gamma$-SV-distributions $\mathbf{r} \leftarrow R$, a better designed mechanism (that they also constructed) is capable of working with all such distributions, provided that the utility $\rho$ is now relaxed to be polynomial in $1 / \varepsilon$, whose degree and coefficients depend on $\gamma$, but not on the size of the database $D$. Moreover, the value $\varepsilon$ can be made an arbitrarily small constant (e.g., $\varepsilon \ll \gamma$ ). This should be contrasted with the impossibility results for the traditional privacy [MP90,DOPS04] with SV sources, where it was shown that $\varepsilon=\Omega(\gamma)$, meaning that even a fixed constant (let alone "negligible") security is impossible. Hence, the result of [DLMV12] suggested a qualitative gap between traditional and differential privacy, but left open the question whether differential privacy is possible with more realistic (i.e., less structured) sources than the SV sources. Indeed, the SV sources seem to be primarily interesting from the perspective of negative results, since real-world distributions are unlikely to produce a sequence of bits, each of which has almost a full unit of fresh entropy.

Our Results In Brief. In part motivated by solving this question, we abstract and generalize prior techniques for showing impossibility results for achieving privacy with various imperfect sources of randomness. Unlike prior work (with the exception of [BD07]), which focused on specific realistic imperfect sources $\mathcal{R}$ (e.g., SV sources), we obtain most of our results for general sources $\mathcal{R}$, but then use various realistic sources (namely, SV sources [SV86], weak/block sources [CG88], and Bias-Control Limited sources [Dod01]) as specific examples to illustrate our technique. In particular, we introduce the concepts of expressiveness and separability of a given imperfect source $\mathcal{R}$ as a measure of its "imperfectness", and show the following results:

- Low levels of expressiveness generically imply strong impossibility results for both traditional and differential privacy.

- Separability implies expressiveness; NON-separability is equivalent to "weak bit extraction".

- While the separability of some concrete (e.g., SV) sources $\mathcal{R}$ was implicitly known, we show new separability results for several important sources, including general "block sources".

We stress that the first two results are completely generic, and reduce the question of feasibility of privacy under $\mathcal{R}$ to a much easier and self-contained question of separability of $\mathcal{R}$. And establishing the latter is the only "source-specific" technical work which remains. In particular, after explicitly stating known separability results for SV and weak sources, and establishing our new separability results for bias-control limited (BCL) and block sources, we obtain the following direct corollaries:

- Existing, but quantitatively improved, impossibility results for traditional privacy, but under a wider variety of sources $\mathcal{R}$ (block, SV, weak, BCL).

- First impossibility results for differential privacy. Although, unsurprisingly, these results (barely) miss the highly structured SV sources, they come back extremely quickly once the source becomes slightly more realistic (e.g., a very "constrained" block/BCL/weak source).

\footnotetext{
${ }^{2}$ So called Laplacian distribution, but the details do not matter here.
} 
- Any imperfect source allowing (either traditional or differential) privacy admits a certain type of deterministic bit extraction. (This result is incomparable to the result of [BD07].)

We briefly expand on these results below, but conclude that, despite the result of [DLMV12], our results seem to unify and strengthen the belief that, for the most part, privacy with imperfect randomness is impossible, unless the source is (almost) deterministically extractable. More importantly, they provide an intuitive, modular and unified picture elucidating the (im)possibility of privacy with general imperfect sources.

\subsection{Our Results in More Detail}

At a high level, our results follow the blueprint of [DOPS04] (who concentrated exclusively on the SV sources), but in significantly more modular and quantitatively optimized way (making our proofs somewhat more illuminating, in our opinion). In essence, they establish an impossibility of a given privacy task $P$ under a source $\mathcal{R}$ using three steps:

STEP 1: PRIVACY TASK $P$ UNDER $\mathcal{R} \longrightarrow$ EXPRESSIVENESS OF $\mathcal{R}$. Intuitively, expressiveness of $\mathcal{R}$ means that $\mathcal{R}$ is rich enough to "distinguish" any functions $f$ and $g$ which are not point-wise equal almost everywhere (see Definition 1): there exists $R \in \mathcal{R}$ s.t. $\mathrm{SD}(f(R), g(R))$ is "noticeable", where SD is the statistical distance between distributions. ${ }^{3}$ With this clean abstraction, we almost trivially show (see Theorem 1) that most traditional privacy tasks $P$ (extraction, encryption, secret sharing, commitment) imply the existence of sufficiently-distinct functions $f$ and $g$ which would violate the expressibility of $\mathcal{R}$. For example, such $f(\mathbf{r})$ and $g(\mathbf{r})$ are simply the encryptions of two different plaintexts under key $\mathbf{r}$ when $P$ is encryption, and similar arguments hold for commitment, extraction and secret sharing schemes.

More interestingly, we show that expressiveness is again sufficient to rule out even differential privacy (Theorem 2). The proof follows the same high-level intuition as for the traditional privacy, but is somewhat more involved. This is because DP only gives us security for "close" databases, while the utility guarantees are only meaningful for "far" databases. In particular, for this reason it will turn out that the expressiveness requirement on $\mathcal{R}$ for ruling out differential privacy will be slightly higher than for traditional privacy (Theorem 2 vs. Theorem 1 ). ${ }^{4}$ Still, aside from this quantitative difference, there is no qualitative difference between our arguments for traditional and differential privacy.

Overall, the deceptive simplicity of our "privacy-to-expressiveness" arguments is actually a feature of our framework, as these arguments are the only place when the specific details of $P$ matter, as the rest of the framework - described below - will only concentrate on the expressiveness of $\mathcal{R}$ !

STEP 2: EXPRESSIVENESS OF $\mathcal{R} \longrightarrow$ SEPARABILITY OF $\mathcal{R}$. Intuitively, separability of $\mathcal{R}$ means that $\mathcal{R}$ is rich enough to "separate" any sufficiently large disjoint sets $G$ and $B$ (see Definition 8; wlog, assume that $|G| \geq|B|)$ : there exists $R \in \mathcal{R}$ s.t. $(\operatorname{Pr}[R \in G]-\operatorname{Pr}[R \in B])$ is "noticeable". ${ }^{5}$ A moment reflection shows that separability is closely related to expressiveness, but restricted to boolean functions $f$ and $g$ of disjoint support (namely, the characteristic functions of $G$ and $B$ ), which makes it noticeably easier to work with (as we will see).

Nevertheless, we show that separability generically implies expressiveness, with nearly identical parameters (see Theorem 3). This is where we differ and quantitatively improve the argument implicit in [DOPS04]: while [DOPS04] used a bit-by-bit hybrid argument to show expressiveness (for the SV source), our proof of Theorem 3 used a more clever "universal hashing trick" ${ }^{6}$ This allowed us to obtain results which are independent of the ranges of $f$ and $g$ (which, in turn, will later correspond to bit sizes of ciphertexts, commitments, secret shares, etc.)

Of independent interest, we also show that NON-separability of $\mathcal{R}$ is equivalent to a certain type of "weak bit extraction" from $\mathcal{R}$ (see Theorem 4): (a) when produced, the extracted bit is guaranteed to be

\footnotetext{
${ }^{3}$ Like in [DOPS04] and unlike [MP90], our distinguishers between $f(R)$ and $g(R)$ will be very efficient, but we will not require this in order not to clutter the notation.

4 Jumping ahead, this will be the reason while our new impossibility results for DP will (barely) miss the SV sources, but will come back very quickly once the source will become more realistic.

${ }^{5}$ For example, if $\mathcal{R}$ only consists of the uniform distribution $U$, the latter is impossible when $|G|=|B|$. In contrast, we will see that all natural "non-extractable" sources (SV, weak, block, BCL) are separable.

${ }^{6}$ Similar trick with randomness extractors was used, in a slightly different context, by $\left[\mathrm{ACM}^{+} 14\right]$.
} 
almost unbiased, (b) although the extractor is allowed to fail, it will typically succeed at least on the uniform distribution. $^{7}$

Coupled with Step 1, we get the following two implications. First, we reduce the impossibility of many privacy tasks $P$ under $\mathcal{R}$ to a much easier question of separability of $\mathcal{R}$ (which is independent of $P$ ). Second, we generically show that the feasibility of $P$ under $\mathcal{R}$ implies deterministic weak bit extraction from $\mathcal{R}$, incomparably complementing the prior result of [BD07]. Namely, [BD07] showed that several traditional privacy primitives, including (only multi-bit) encryption and commitment (but not secret sharing) imply the existence of multi-bit deterministic extraction schemes capable of extracting almost the same number of bits as the plaintext. On the positive, our result applies to a much wider set of primitives $P$ (e.g., secret-sharing, as well as even single-bit encryption and commitment). On the negative, we can only argue a rather weak kind of single-bit extraction, where the extractor is allowed to fail, while [BD07] showed traditional, and possibly multi-bit, extraction.

STEP 3: SEPARABILITY OF VARIOUS SOURCES $\mathcal{R}$. Unlike the prior results in [MP90,DOPS04,ACM $\left.{ }^{+} 14\right]$, all the above results are true for any imperfect source $\mathcal{R}$. To get concrete impossibility results for natural sources, though, we finally must establish good separability bounds for specific $\mathcal{R}$. Such bounds were already implicitly known [DOPS04] (or trivial to see) for the SV and general weak sources, but we show how they can be also demonstrated for other natural sources: block sources [CG88] and bias-control-limited (BCL) sources [Dod01]. In particular, our separability bounds for block sources turned out to be quite non-trivial, and form one of the more technical contributions of this work. See the proof of Lemma 2(b).

Aside from being natural and interesting it their own right, the new separability results for block/BCL sources are especially interesting from the perspective of differential privacy (see below). Indeed, both of them can be viewed as realistic relaxations of highly-structured SV sources, but yet not as general/unstructured as weak sources. And since we already know that DP is possible with SV sources [DLMV12], it is interesting to know how soon it will take for the impossibility results to come back, once the source slowly becomes more realistic/unstructured, but before going "all the way" to being weak.

Putting it all together: New and Old Impossibility Results. Applying Steps 1-3 to specific sources of interest (weak, block, SV, BCL), we immediately derive a variety of impossibility results for traditional privacy (see Table 1). Although these results were derived mainly as a "warm-up" to our (completely new) impossibility results for differentially privacy, they offer quantitative improvements to the results of [DOPS04] (due to stronger expressiveness-to-separability reduction). For example, they rule out even constant (as opposed to negligible) security for encryption/commitment/secret sharing, irrespective of the sizes of ciphertexts/commitments/shares. Related, we unsurprisingly get stronger impossibility results for BCL/block sources than the more structured SV sources.

More interestingly, we obtain first impossibility results for differential privacy with imperfect randomness. In light of the positive result of [DLMV12], our separability result for SV sources is (barely) not strong enough to rule out differential privacy under SV sources. As we explained, this failure happened not because our framework was too weak to apply to SV sources or differential privacy, but rather due to a "local-vs-global gap" between the privacy and utility requirements for differential privacy.

However, once we consider general weak sources, or even much more structured BCL/block sources, the impossibility results come back extremely quickly! For example, when studying $\varepsilon$-DP with utility $\rho, n$-bit weak sources of min-entropy $k$ are ruled out the moment $k=n-\log (\varepsilon \rho)-O(1)$ (Theorem $6(\mathrm{a})),{ }^{8}$ while BCL sources are ruled out the moment the number of "SV bits" $b$ the attacker can fix completely (instead of only bias by $\gamma$ ) is just $b=\Omega(\log (\varepsilon \rho) / \gamma$ ) (Theorem $6(\mathrm{c})$ ). As $\varepsilon \rho$ is typically desired to be a constant, $\log (\varepsilon \rho)$ is an even smaller constant, which means we even rule out constant entropy deficiency $(n-k)$ (or $m-k$ for block source) or number of "interventions" $b$, respectively. We also compare impossibility results for traditional and differential privacy in Table 2, and observe that the latter are only marginally weaker than the former. This leads us to the conclusion that differential privacy is still rather demanding to achieve with realistic imperfect sources of randomness.

\footnotetext{
${ }^{7}$ Unfortunately, we demonstrate that the limitation of part (b) holding only for the uniform distribution is somewhat inherent in this great level of generality.

8 More generally, even $n$-bit block sources with block length $m$ and fresh min-entropy $k$ per block are ruled out when $k=m-\log (\varepsilon \rho)-O(1)$, irrespective of the number of blocks $n / m$. See Theorem $6(\mathrm{~b})$.
} 


\section{Preliminaries}

For a positive integer $n$, let $[n] \stackrel{\text { def }}{=}\{1,2, \ldots, n\}$. For a set $S$, we write $U_{S}$ to denote the uniform distribution over $S$. For simplicity, denote $U_{n} \stackrel{\text { def }}{=} U_{\{0,1\}^{n}}$. For a distribution or a random variable $R$, let $\mathbf{r} \leftarrow R$ denote the operation of sampling a random $\mathbf{r}$ according to $R$. We call a family of distributions over $\{0,1\}^{n}$ a source, denoted as $\mathcal{R}_{n}$. All logarithms are to the base 2. The min-entropy of a random variable $R$ is defined as $\mathbf{H}_{\infty}(R) \stackrel{\text { def }}{=} \min _{\mathbf{r} \in \operatorname{supp}(R)} \log \frac{1}{\operatorname{Pr}[R=\mathbf{r}]}$.

For two random variables $R$ and $R^{\prime}$ over $\{0,1\}^{n}$, the statistical distance between $R$ and $R^{\prime}$ is denoted as $\mathrm{SD}\left(R, R^{\prime}\right) \stackrel{\text { def }}{=} \frac{1}{2} \sum_{\mathbf{r} \in\{0,1\}^{n}}\left|\operatorname{Pr}[R=\mathbf{r}]-\operatorname{Pr}\left[R^{\prime}=\mathbf{r}\right]\right|=\max _{\operatorname{Eve}}\left|\operatorname{Pr}[\operatorname{Eve}(R)=1]-\operatorname{Pr}\left[\operatorname{Eve}\left(R^{\prime}\right)=1\right]\right|$, where each Eve is a distinguisher. We say that the relative distance between $R$ and $R^{\prime}$ is $\varepsilon$, denoted as $\operatorname{RD}\left(R, R^{\prime}\right)=\varepsilon$, if $\varepsilon$ is the smallest number such that $\operatorname{Pr}[R=\mathbf{r}] \in\left[e^{-\varepsilon} \cdot \operatorname{Pr}\left[R^{\prime}=\mathbf{r}\right], e^{\varepsilon} \cdot \operatorname{Pr}\left[R^{\prime}=\mathbf{r}\right]\right]$ for all $\mathbf{r} \in\{0,1\}^{n}$. It's easy to see that $\mathrm{RD}\left(R, R^{\prime}\right) \leq \varepsilon$ implies $\mathrm{SD}\left(R, R^{\prime}\right) \leq e^{\varepsilon}-1$.

\section{Expressiveness and its Implications to Privacy}

In this section, we introduce the concept of expressiveness of a source. Then we study its implications to traditional and differential privacy.

Informally, an expressive source $\mathcal{R}_{n}$ can separate two distributions $f(R)$ and $g(R)$, unless the functions $f$ and $g$ are point-wise equal almost everywhere.

Definition 1. We call that a source $\mathcal{R}_{n}$ is $(t, \delta)$-expressive if for any functions $f, g:\{0,1\}^{n} \rightarrow \mathcal{C}$, where $\mathcal{C}$ is any universe, such that $\operatorname{Pr}_{\mathbf{r} \leftarrow U_{n}}[f(\mathbf{r}) \neq g(\mathbf{r})] \geq \frac{1}{2^{t}}$ for some $t \geq 0$, there exists a distribution $R \in \mathcal{R}_{n}$ such that $S D(f(R), g(R)) \geq \delta$.

\subsection{Implications to Traditional Privacy}

We recall (or define) some cryptographic primitives related to traditional privacy: bit extractor, bit encryption scheme, weak bit commitment, and bit $T$-secret sharing as follows.

Definition 2. We say that Ext $:\{0,1\}^{n} \rightarrow\{0,1\}$ is $\left(\mathcal{R}_{n}, \delta\right)$-secure bit extractor if for every distribution $R \in \mathcal{R}_{n},\left|\operatorname{Pr}_{\mathbf{r} \leftarrow R}[\operatorname{Ext}(\mathbf{r})=1]-\operatorname{Pr}_{\mathbf{r} \leftarrow R}[\operatorname{Ext}(\mathbf{r})=0]\right|<\delta$ (equivalently, $S D\left(\operatorname{Ext}(R), U_{1}\right)<\delta / 2$ ).

In the following, we consider the simplest encryption scheme, where the plaintext is composed of a single bit $x$.

Definition 3. $A\left(\mathcal{R}_{n}, \delta\right)$-secure bit encryption scheme is a tuple of functions Enc : $\{0,1\}^{n} \times\{0,1\} \rightarrow\{0,1\}^{\lambda}$ and Dec: $\{0,1\}^{n} \times\{0,1\}^{\lambda} \rightarrow\{0,1\}$, where, for convenience, $\operatorname{Enc}(\mathbf{r}, x)$ (resp. $\operatorname{Dec}(\mathbf{r}, \mathbf{c})$ ) is denoted as $\operatorname{Enc}_{\mathbf{r}}(x)$ (resp. Dec $\mathbf{r}(\mathbf{c}))$, satisfying the following two properties:

(a) Correctness: for all $\mathbf{r} \in\{0,1\}^{n}$ and $x \in\{0,1\}, \operatorname{Dec}_{\mathbf{r}}\left(\operatorname{Enc}_{\mathbf{r}}(x)\right)=x$;

(b) Statistical Hiding: $S D\left(E n c_{R}(0), E n c_{R}(1)\right)<\delta$, for every distribution $R \in \mathcal{R}_{n}$.

Commitment schemes allow the sender Alice to commit a chosen value (or statement) while keeping it secret from the receiver Bob, with the ability to reveal the committed value in a later stage. Binding and hiding properties are essential to any commitment scheme. Informally,

- Binding: it is "hard" for Alice to alter her commitment after she has made it;

- Hiding: it is "hard" for Bob to find out the committed value without Alice revealing it.

Each of them can be computational or information theoretical. However, we can't achieve information theoretically binding and information theoretically hiding properties at the same time. Instead of defining computational notions, we relax binding to some very weak property, so that hiding and this new (very weak) binding properties both can be information theoretical. Since we aim to show an impossibility result, such relaxation is justified. 
Definition 4. $A\left(\mathcal{R}_{n}, \delta\right)$-secure weak bit commitment is a function Com $:\{0,1\}^{n} \times\{0,1\} \rightarrow\{0,1\}^{\lambda}$ satisfying the following two properties:

(a) Weak Binding: $\underset{\mathbf{r} \leftarrow U_{n}}{\operatorname{Pr}}[\operatorname{Com}(0 ; \mathbf{r}) \neq \operatorname{Com}(1 ; \mathbf{r})] \geq \frac{1}{2}$;

(b) Statistical Hiding: $\operatorname{SD}(\operatorname{Com}(0 ; R), \operatorname{Com}(1 ; R))<\delta$, for every distribution $R \in \mathcal{R}_{n}$.

Note that in the traditional notion of commitment, the binding property holds if it is "hard" to find $\mathbf{r}_{1}$ and $\mathbf{r}_{2}$ such that $\operatorname{Com}\left(0 ; \mathbf{r}_{1}\right)=\operatorname{Com}\left(1 ; \mathbf{r}_{2}\right)$. Here we give a much weaker binding notion. We only require that the attacker can not win with probability $\geq \frac{1}{2}$ by choosing $\mathbf{r}_{1}=\mathbf{r}_{2}$ uniformly at random. For example, $\operatorname{Com}(x ; r)=x \oplus r$, where $x, r \in\{0,1\}$ can be easily verified to be a weak bit commitment for any $\delta>0$ (despite not being a standard commitment).

In the notion of $T$-party Secret Sharing, two thresholds $T_{1}$ and $T_{2}$, where $1 \leq T_{1}<T_{2} \leq T$, are involved such that (a) any $T_{1}$ parties have "no information" about the secret, (b) any $T_{2}$ parties enable to recover the secret. Because our purpose is to show an impossibility result, we restrict to $T_{1}=1$ and $T_{2}=T$, and only consider one bit secret $x$.

Definition 5. A $\left(\mathcal{R}_{n}, \delta\right)$-secure bit $T$-Secret Sharing scheme is a tuple (Share, Share $2, \ldots$, Share $_{T}$, Rec) satisfying the following two properties:

(a) Correctness: for all $\mathbf{r} \in\{0,1\}^{n}$ and $x \in\{0,1\}, \operatorname{Rec}\left(\operatorname{Share}_{1}(x, \mathbf{r}), \operatorname{Share}_{2}(x, \mathbf{r}), \ldots, \operatorname{Share}_{T}(x, \mathbf{r})\right)=x$;

(b) Statistical Hiding: $\operatorname{SD}\left(\operatorname{Share}_{j}(0 ; R)\right.$, Share $\left._{j}(1 ; R)\right)<\delta$, for every index $j \in[T]$ and distribution $R \in \mathcal{R}_{n}$.

Now we abstract and generalize the results of [MP90,DOPS04] to show that expressiveness implies the impossibility of security involving traditional privacy.

\section{Theorem 1.}

(a) If a source $\mathcal{R}_{n}$ is $(0, \delta)$-expressive, then no $\left(\mathcal{R}_{n}, \delta\right)$-secure bit extractor exists.

(b) If a source $\mathcal{R}_{n}$ is $(0, \delta)$-expressive, then no $\left(\mathcal{R}_{n}, \delta\right)$-secure bit encryption scheme exists.

(c) If a source $\mathcal{R}_{n}$ is $(1, \delta)$-expressive, then no $\left(\mathcal{R}_{n}, \delta\right)$-secure weak bit commitment exists.

(d) If a source $\mathcal{R}_{n}$ is $(\log T, \delta)$-expressive, then no $\left(\mathcal{R}_{n}, \delta\right)$-secure bit $T$-secret sharing exists.

Proof.

(a) Assume that there exists a $\left(\mathcal{R}_{n}, \delta\right)$-secure bit extractor Ext. Define $f(\mathbf{r}) \stackrel{\text { def }}{=} \operatorname{Ext}(\mathbf{r})$ and $g(\mathbf{r}) \stackrel{\text { def }}{=} 1-\operatorname{Ext}(\mathbf{r})$. Since for all $\mathbf{r} \in\{0,1\}^{n}$, it holds that $\operatorname{Ext}(\mathbf{r}) \neq 1-\operatorname{Ext}(\mathbf{r})$, we get $\operatorname{Pr}_{\mathbf{r} \leftarrow U_{n}}[f(\mathbf{r}) \neq g(\mathbf{r})]=1=\frac{1}{2^{0}}$. Definition 1 implies that there exists a distribution $R \in \mathcal{R}_{n}$ such that $\mathrm{SD}(f(R), g(R)) \geq \delta$. Therefore,

$$
|\operatorname{Pr}[\operatorname{Ext}(R)=1]-\operatorname{Pr}[\operatorname{Ext}(R)=0]|=\mathrm{SD}(f(R), g(R)) \geq \delta,
$$

which is a contradiction.

(b) Assume that there exists a $\left(\mathcal{R}_{n}, \delta\right)$-secure bit encryption scheme. Define $f(\mathbf{r}) \stackrel{\text { def }}{=} \operatorname{Enc}_{\mathbf{r}}(0)$ and $g(\mathbf{r}) \stackrel{\text { def }}{=}$ $\operatorname{Enc}_{\mathbf{r}}(1)$. Since for all secret keys $\mathbf{r} \in\{0,1\}^{n}$, it holds that $\operatorname{Enc}_{\mathbf{r}}(0) \neq \operatorname{Enc}_{\mathbf{r}}(1)$, we have $\underset{\mathbf{r} \leftarrow U_{n}}{\operatorname{Pr}}[f(\mathbf{r}) \neq g(\mathbf{r})]=$ $1=\frac{1}{2^{0}}$. Definition 1 implies that there exists a distribution $R \in \mathcal{R}_{n}$ such that $\operatorname{SD}(f(R), g(R)) \geq \delta$, which is in contradiction to $\mathrm{SD}(f(R), g(R))<\delta$.

(c) Assume that there exists a $\left(\mathcal{R}_{n}, \delta\right)$-secure weak bit commitment. Define $f(\mathbf{r}) \stackrel{\text { def }}{=} \operatorname{Com}(0 ; \mathbf{r})$ and $g(\mathbf{r}) \stackrel{\text { def }}{=}$ $\operatorname{Com}(1 ; \mathbf{r})$. Since $\underset{\mathbf{r} \leftarrow U_{n}}{\operatorname{Pr}}[\operatorname{Com}(0 ; \mathbf{r}) \neq \operatorname{Com}(1 ; \mathbf{r})] \geq \frac{1}{2}$, there exists a distribution $R \in \mathcal{R}_{n}$ such that $\mathrm{SD}(f(R), g(R))$ $\geq \delta$, which is in contradiction to $\mathrm{SD}(f(R), g(R))<\delta$.

(d) Assume that there exists a $\left(\mathcal{R}_{n}, \delta\right)$-secure bit $T$-secret sharing. Let $t=\log T$. Then for all $\mathbf{r} \in\{0,1\}^{n}$,

$$
\begin{aligned}
& \text { Share } \left._{1}(0 ; \mathbf{r}), \text { Share }_{2}(0 ; \mathbf{r}), \ldots, \text { Share }_{T}(0 ; \mathbf{r})\right) \neq\left(\operatorname{Share}_{1}(1 ; \mathbf{r}), \operatorname{Share}_{2}(1 ; \mathbf{r}), \ldots, \text { Share }_{T}(1 ; \mathbf{r})\right) \\
& \Rightarrow \text { there exists } j=j(\mathbf{r}) \text { such that } \operatorname{Share}_{j}(0 ; \mathbf{r}) \neq \operatorname{Share}_{j}(1 ; \mathbf{r}) . \\
& \Rightarrow \text { there exists } j^{*} \in[T] \text { such that }\left|\left\{\mathbf{r} \mid j(\mathbf{r})=j^{*}\right\}\right| \geq \frac{2^{n}}{T}=2^{n-t}
\end{aligned}
$$


Define $f(\mathbf{r}) \stackrel{\text { def }}{=}$ Share $j^{*}(0 ; \mathbf{r})$ and $g(\mathbf{r}) \stackrel{\text { def }}{=} \operatorname{Share}_{j^{*}}(1 ; \mathbf{r})$. Then $\underset{\mathbf{r} \leftarrow U_{n}}{\operatorname{Pr}_{n}}[f(\mathbf{r}) \neq g(\mathbf{r})] \geq \frac{1}{2^{t}}$. Therefore, there exists a distribution $R \in \mathcal{R}_{n}$ such that $\mathrm{SD}(f(R), g(R)) \geq \delta$, which is in contradiction to $\mathrm{SD}(f(R), g(R))<\delta$.

\subsection{Implications to Differential Privacy}

Dodis et al. [DLMV12] have shown how to do differential privacy with respect to the $\gamma$-SV source for all "queries of low sensitivity". Since we aim to show impossibility results, henceforth we only consider the simplest case: let $\mathcal{D}=\{0,1\}^{N}$ be the space of all databases and for $D \in \mathcal{D}$, the query function $q$ is the Hamming weight function $w t(D)=|\{i \mid D(i)=1\}|$, where $D(i)$ means the $i$-th bit ("record") of $D$. If the source $\mathcal{R}_{n}$ has only one distribution $U_{n}$, denote $\mathcal{R}_{n}$ as $U_{n}$ for simplicity. For any $D, D^{\prime} \in \mathcal{D}$, the discrete distance function between them is defined by $\Delta\left(D, D^{\prime}\right) \stackrel{\text { def }}{=} w t\left(D \oplus D^{\prime}\right)$, where $\oplus$ is the bitwise exclusive OR operator. We say that two databases $D$ and $D^{\prime}$ are neighboring if $\Delta\left(D, D^{\prime}\right)=1$. A mechanism $M$ is an algorithm that takes as input a database $D \in \mathcal{D}$ and a random variable $R \in \mathcal{R}_{n}$, and outputs a random value $z$. Informally, we wish $z=M(D, R)$ to approximate the true Hamming weight $w t(D)$ without revealing too much information about any individual $D(i)$. More formally, a mechanism is differentially private for the Hamming weight queries if replacing an entry in the database with one containing fake information only changes the output distribution of the mechanism by a small amount. In other words, evaluating the mechanism on two neighboring databases, does not change the outcome distribution by much. On the other hand, we define its utility to be the expected difference between the true answer $w t(D)$ and the output of the mechanism. More formally,

Definition 6. Let $\varepsilon \geq 0$ and $\mathcal{R}_{n}$ be a source. A mechanism $M$ (for the Hamming weight queries) is $\left(\mathcal{R}_{n}, \varepsilon\right.$ )differentially private if for all neighboring databases $D_{1}, D_{2} \in \mathcal{D}$, and all distributions $R \in \mathcal{R}_{n}$, we have $R D\left(M\left(D_{1}, R\right), M\left(D_{2}, R\right)\right) \leq \varepsilon$. Equivalently, for any possible output $z$ :

$$
\underset{\mathbf{r} \leftarrow R}{\left.\left.\frac{\operatorname{Pr}_{\mathbf{r} \leftarrow R}}{\operatorname{Pr}_{R}\left[M\left(D_{1}, \mathbf{r}\right)=z\right]} \leq D_{2}, \mathbf{r}\right)=z\right]} \leq e^{\varepsilon} .
$$

We also note that for $\varepsilon<1$, we can rather accurately approximate $e^{\varepsilon}$ by $1+\varepsilon$.

Definition 7. Let $0<\rho \leq N / 4$ and $\mathcal{R}_{n}$ be a source. A mechanism $M$ has $\left(\mathcal{R}_{n}, \rho\right)$-utility for the Hamming weight queries, if for all databases $D \in \mathcal{D}$ and all distributions $R \in \mathcal{R}_{n}$, we have:

$$
\mathbb{E}_{\mathbf{r} \leftarrow R}[|M(D, \mathbf{r})-w t(D)|] \leq \rho .
$$

We show that, much like with traditional privacy, expressiveness implies impossibility of differential privacy with imperfect randomness, albeit with slightly more demanding parameters. As a high-level idea, for two databases $D$ and $D^{\prime}$, define two functions $f(\mathbf{r}) \stackrel{\text { def }}{=} M(D, \mathbf{r})$ and $g(\mathbf{r}) \stackrel{\text { def }}{=} M\left(D^{\prime}, \mathbf{r}\right)$. Intuitively, for all $R \in \mathcal{R}_{n}$, since $\operatorname{RD}(f(R), g(R)) \leq \varepsilon \cdot \Delta\left(D, D^{\prime}\right)$ implies $\mathrm{SD}(f(R), g(R)) \leq e^{\varepsilon \cdot \Delta\left(D, D^{\prime}\right)}-1$, we could use expressiveness to argue that $f(\mathbf{r})=g(\mathbf{r})$ almost everywhere, which must eventually contradict utility (even for uniform distribution). However, we can't use this technique directly, because if $\varepsilon \cdot \Delta\left(D, D^{\prime}\right)$ is large enough, then $e^{\varepsilon \cdot \Delta\left(D, D^{\prime}\right)}-1>1$, which is greater than the general upper bound 1 of the statistical distance. Instead, we simply use this trick on close-enough databases $D$ and $D^{\prime}$, and then use a few "jumps" from $D_{0}$ to $D_{1}$, etc., until eventually we must violate the $\rho$-utility. Details follow.

Theorem 2. Assume $1 /(8 \rho) \leq \varepsilon \leq 1 / 4$ and the source $\mathcal{R}_{n}$ is $\left(\log \left(\frac{\rho \varepsilon}{\delta}\right)+4, \delta\right)$-expressive, for some $2 \varepsilon \leq \delta \leq 1$. Then no $\left(\mathcal{R}_{n}, \varepsilon\right)$-differentially private and $\left(U_{n}, \rho\right)$-accurate mechanism for the Hamming weight queries exists. In particular, plugging $\delta=2 \varepsilon$ and $\delta=\frac{1}{2}$, respectively, this holds if either

(a) $\mathcal{R}_{n}$ is $(3+\log (\rho), 2 \varepsilon)$-expressive; or

(b) $\mathcal{R}_{n}$ is $\left(5+\log (\rho \varepsilon), \frac{1}{2}\right)$-expressive. 
Proof. Assume for contradiction that there exists such a mechanism $M$. Let $\mathcal{D}^{\prime} \stackrel{\text { def }}{=}\{D \mid w t(D) \leq 4 \rho\}$. Denote

$$
\operatorname{Trunc}(x) \stackrel{\text { def }}{=}\left\{\begin{aligned}
0, & \text { if } x<0 \\
x, & \text { if } x \in\{0,1, \ldots, 4 \rho\} \\
4 \rho, & \text { otherwise. }
\end{aligned}\right.
$$

For any $D \in \mathcal{D}^{\prime}$, define the truncated mechanism $M^{\prime} \stackrel{\text { def }}{=} \operatorname{Trunc}(M)$ by $M^{\prime}(D, \mathbf{r}) \stackrel{\text { def }}{=} \operatorname{Trunc}(M(D, \mathbf{r}))$. Since for every $D \in \mathcal{D}^{\prime}$, we have $w t(D) \in\{0,1, \ldots, 4 \rho\}, M^{\prime}$ still has $\left(U_{n}, \rho\right)$-utility on $\mathcal{D}^{\prime}$. Additionally, from Definition 6, it's straightforward that $M^{\prime}$ is $\left(\mathcal{R}_{n}, \varepsilon\right)$-differentially private on $\mathcal{D}^{\prime}$. In the following, we only consider the truncated mechanism $M^{\prime}$ on $\mathcal{D}^{\prime}$.

Let $t=\log \left(\frac{\rho \varepsilon}{\delta}\right)+4$ and $s=\frac{\delta}{2 \varepsilon}$. Notice, $1 \leq s \leq 1 /(2 \varepsilon) \leq 4 \rho, e^{\varepsilon s}-1<\delta$, and $2^{t}=8 \rho / s$.

We start with the following claim:

Claim. Consider any databases $D, D^{\prime} \in \mathcal{D}^{\prime}$, s.t. $\Delta\left(D, D^{\prime}\right) \leq s$, and denote $f(\mathbf{r}) \stackrel{\text { def }}{=} M^{\prime}(D, \mathbf{r})$ and $g(\mathbf{r}) \stackrel{\text { def }}{=}$ $M^{\prime}\left(D^{\prime}, \mathbf{r}\right)$. Then $\operatorname{Pr}_{\mathbf{r} \leftarrow U_{n}}[f(\mathbf{r}) \neq g(\mathbf{r})]<\frac{1}{2^{t}}$.

Proof. Since $M^{\prime}$ is $\left(\mathcal{R}_{n}, \varepsilon\right)$-differentially private, then for all $R \in \mathcal{R}_{n}$, we have $\operatorname{RD}(f(R), g(R)) \leq \varepsilon$. $\Delta\left(D, D^{\prime}\right) \leq \varepsilon \cdot s$. Hence, $\operatorname{SD}(f(R), g(R)) \leq e^{\varepsilon \cdot s}-1<\delta$, by our choice of $s$. Since this holds for all $R \in \mathcal{R}_{n}$ and $\mathcal{R}_{n}$ is $(t, \delta)$-expressive, we conclude that it must be the case that $\operatorname{Pr}_{\mathbf{r} \leftarrow U_{n}}[f(\mathbf{r}) \neq g(\mathbf{r})]<\frac{1}{2^{t}}$.

Coming back to the main proof, consider a sequence of databases $D_{0}, D_{1}, \cdots, D_{4 \rho / s}$ such that $w t\left(D_{i}\right)=$ $i \cdot s$ and $\Delta\left(D_{i}, D_{i+1}\right)=s$. Denote $f_{i}(R) \stackrel{\text { def }}{=} M^{\prime}\left(D_{i}, R\right)$ for all $i \in\{0,1, \ldots, 4 \rho / s\}$. From the above Claim, we get that $\underset{\mathbf{r} \leftarrow U_{n}}{\operatorname{Pr}}\left[f_{i}(\mathbf{r}) \neq f_{i+1}(\mathbf{r})\right]<\frac{1}{2^{t}}$. By the union bound and our choice of $s$ and $t$,

$$
\operatorname{Pr}_{\mathbf{r} \leftarrow U_{n}}\left[f_{0}(\mathbf{r}) \neq f_{4 \rho / s}(\mathbf{r})\right]<\frac{4 \rho}{2^{t} \cdot s} \leq \frac{1}{2}
$$

Let $\alpha \stackrel{\text { def }}{=} \mathbb{E}_{\mathbf{r} \leftarrow U_{n}}\left[f_{4 \rho / s}(\mathbf{r})-f_{0}(\mathbf{r})\right]$. From $\left(U_{n}, \rho\right)$-utility, we get that

$$
\alpha \geq\left(w t\left(D_{4 \rho / s}\right)-\rho\right)-\left(w t\left(D_{0}\right)+\rho\right)=(4 \rho-\rho)-(0+\rho)=2 \rho .
$$

On the other hand, from Inequation (1),

$$
\alpha \leq \operatorname{Pr}_{\mathbf{r} \leftarrow U_{n}}\left[f_{0}(\mathbf{r}) \neq f_{4 \rho / s}(\mathbf{r})\right] \cdot \max _{\mathbf{r}}\left|f_{4 \rho / s}(\mathbf{r})-f_{0}(\mathbf{r})\right|<\frac{1}{2} \cdot 4 \rho=2 \rho,
$$

which is a contradiction.

\section{$4 \quad$ Separability and its Implications}

Expressiveness is a powerful tool, but it's hard for us to use it directly. In this section, we introduce the concept of separability and show it implies expressiveness, and also has its own applications to (weak) coin flipping. Several typical examples can been seen in Section 5.

Intuitively, separable sources $\mathcal{R}_{n}$ allow one to choose a distribution $R \in \mathcal{R}_{n}$ capable of "separating" any sufficiently large, disjoint sets $G$ and $B$ : increasing a relative weight of one set w.r.t. $R$ without doing the same for the counterpart of the other one.

Definition 8. We say that a source $\mathcal{R}_{n}$ is $(t, \delta)$-separable if for all $G, B \subseteq\{0,1\}^{n}$, where $G \cap B=\emptyset$ and $|G \cup B| \geq 2^{n-t}$, there exists a distribution $R \in \mathcal{R}_{n}$ such that

$$
\left|\operatorname{Pr}_{\mathbf{r} \leftarrow R}[\mathbf{r} \in G]-\operatorname{Pr}_{\mathbf{r} \leftarrow R}[\mathbf{r} \in B]\right| \geq \delta .
$$




\subsection{Separability Implies Expressiveness}

We investigate the relationship between separability and expressiveness. We show that separable sources must be expressive. The high-level idea of the proof comes from the work of [DOPS04] (who only applied it to SV sources), but we quantitatively improve the technique of [DOPS04], by making the gap between expressiveness and separability independent of the range $\mathcal{C}$ of the functions $f$ and $g$.

Theorem 3. If a source $\mathcal{R}_{n}$ is $(t+1, \delta)$-separable, then it's $(t, \delta)$-expressive.

Proof. Suppose that $f, g:\{0,1\}^{n} \rightarrow \mathcal{C}$ are two arbitrary functions such that $\operatorname{Pr}_{\mathbf{r} \leftarrow U_{n}}[f(\mathbf{r}) \neq g(\mathbf{r})] \geq \frac{1}{2^{t}}$. Let $S=\left\{\mathbf{r} \in\{0,1\}^{n} \mid f(\mathbf{r}) \neq g(\mathbf{r})\right\}$. By assumption, $|S| \geq 2^{n-t}$.

To build intuition, let's start with the special case where $\mathcal{C}=\{0,1\}$, in which case we will even show that $(t, \delta)$-separability is enough (i.e., no need to increase $t$ by 1 ). For $\alpha, \beta \in\{0,1\}$, denote $S_{\alpha \beta}=\left\{\mathbf{r} \in\{0,1\}^{n} \mid\right.$ $f(\mathbf{r})=\alpha$ and $g(\mathbf{r})=\beta\}$.

The distinguisher Eve is defined as Eve $(x)=1 \Leftrightarrow x=0$. Without loss of generality, assume that $\left|S_{01}\right| \geq\left|S_{10}\right|$. Denote $G \stackrel{\text { def }}{=} S_{01}$ and $B \stackrel{\text { def }}{=} S_{10}$. Since $\mathcal{R}_{n}$ is $(t, \delta)$-separable and $|G \cup B| \geq 2^{n-t}$, there exists a distribution $R \in \mathcal{R}_{n}$ such that $\left|\operatorname{Pr}_{\mathbf{r} \leftarrow R}[\mathbf{r} \in G]-\operatorname{Pr}_{\mathbf{r} \leftarrow R}[\mathbf{r} \in B]\right| \geq \delta$. That is, $\left|\operatorname{Pr}_{\mathbf{r} \leftarrow R}\left[\mathbf{r} \in S_{01}\right]-\underset{\mathbf{r} \leftarrow R}{\operatorname{Pr}}\left[\mathbf{r} \in S_{10}\right]\right| \geq \delta$. Therefore,

$$
\begin{aligned}
\mathrm{SD}(f(R), g(R)) & \geq\left|\operatorname{Pr}_{\mathbf{r} \leftarrow R}[\operatorname{Eve}(f(\mathbf{r}))=1]-\operatorname{Pr}_{\mathbf{r} \leftarrow R}[\operatorname{Eve}(g(\mathbf{r}))=1]\right| \\
& =\left|\operatorname{Pr}_{\mathbf{r} \leftarrow R}[f(\mathbf{r})=0]-\operatorname{Pr}_{\mathbf{r} \leftarrow R}[g(\mathbf{r})=0]\right| \\
& =\left|\left\{\operatorname{Pr}_{\mathbf{r} \leftarrow R}\left[\mathbf{r} \in S_{00}\right]+\operatorname{Pr}_{\mathbf{r} \leftarrow R}\left[\mathbf{r} \in S_{01}\right]\right\}-\left\{\operatorname{Pr}_{\mathbf{r} \leftarrow R}\left[\mathbf{r} \in S_{00}\right]+\operatorname{Pr}_{\mathbf{r} \leftarrow R}\left[\mathbf{r} \in S_{10}\right]\right\}\right| \\
& =\left|\operatorname{Pr}_{\mathbf{r} \leftarrow R}\left[\mathbf{r} \in S_{01}\right]-\operatorname{Pr}_{\mathbf{r} \leftarrow R}\left[\mathbf{r} \in S_{10}\right]\right| \\
& \geq \delta .
\end{aligned}
$$

In the following, we analyze the general case. We'll need to use the notion of universal hash function family [CW79] with a single bit output. Recall that $\mathcal{H}=\{h \mid h: \mathcal{C} \rightarrow\{0,1\}\}$ is a family of universal hash functions if for all $z \neq z^{\prime}, \underset{h \leftarrow U_{\mathcal{H}}}{\operatorname{Pr}}\left[h(z) \neq h\left(z^{\prime}\right)\right]=\frac{1}{2}$. Such families are known to exist for any universe $\mathcal{C}$ and can be made efficient in $n$ if $\mathcal{C} \subseteq\{0,1\}^{\text {poly }(n)}$.

For $\alpha, \beta \in\{0,1\}$ and $h \in \mathcal{H}$, denote $S_{\alpha \beta}(h)=\{\mathbf{r} \in S \mid h(f(\mathbf{r}))=\alpha$ and $h(g(\mathbf{r}))=\beta\}$. Then

$$
\begin{aligned}
\mathbb{E}_{h \leftarrow U_{\mathcal{H}}}\left[\left|S_{01}(h)\right|+\left|S_{10}(h)\right|\right] & =\mathbb{E}_{h \leftarrow U_{\mathcal{H}}}\left[\sum_{\mathbf{r} \in S} \chi_{S_{01}(h) \cup S_{10}(h)}(\mathbf{r})\right] \\
& =\sum_{\mathbf{r} \in S} \operatorname{Pr}_{h \leftarrow U_{\mathcal{H}}}\left[\mathbf{r} \in S_{01}(h) \cup S_{10}(h)\right] \\
& =\sum_{\mathbf{r} \in S} \operatorname{Pr}_{h \leftarrow U_{\mathcal{H}}}[h(f(\mathbf{r})) \neq h(g(\mathbf{r}))] \\
& =\frac{|S|}{2},
\end{aligned}
$$

where $\chi_{S_{01}(h) \cup S_{10}(h)}$ denotes the characteristic function of the set $S_{01}(h) \cup S_{10}(h)$.

Hence, there exists a fixed hash function $h^{*} \in \mathcal{H}$ such that $\left|S_{01}\left(h^{*}\right) \cup S_{10}\left(h^{*}\right)\right| \geq \frac{|S|}{2} \geq 2^{n-t-1}$.

Eve is defined as Eve $(C)=1 \Leftrightarrow h^{*}(C)=0$, for all $C \in \mathcal{C}$. Without loss of generality, assume that $\left|S_{01}\left(h^{*}\right)\right| \geq\left|S_{10}\left(h^{*}\right)\right|$. Denote $G \stackrel{\text { def }}{=} S_{01}\left(h^{*}\right)$ and $B \stackrel{\text { def }}{=} S_{10}\left(h^{*}\right)$. Since $\mathcal{R}_{n}$ is $(t+1, \delta)$-separable, there exists a distribution $R^{\prime} \in \mathcal{R}_{n}$ such that $\left|\operatorname{Pr}_{\mathbf{r} \leftarrow R^{\prime}}[\mathbf{r} \in G]-\operatorname{Pr}_{\mathbf{r} \leftarrow R^{\prime}}[\mathbf{r} \in B]\right| \geq \delta$. That is, $\mid \underset{\mathbf{r} \leftarrow R^{\prime}}{\operatorname{Pr}}\left[\mathbf{r} \in S_{01}\left(h^{*}\right)\right]-\operatorname{Pr}_{\mathbf{r} \leftarrow R^{\prime}}[\mathbf{r} \in$ 
$\left.S_{10}\left(h^{*}\right)\right] \mid \geq \delta$. Hence,

$$
\begin{aligned}
\operatorname{SD}\left(f\left(R^{\prime}\right), g\left(R^{\prime}\right)\right) & \geq\left|\operatorname{Pr}_{\mathbf{r} \leftarrow R^{\prime}}[\operatorname{Eve}(f(\mathbf{r}))=1]-\operatorname{Pr}_{\mathbf{r} \leftarrow R^{\prime}}[\operatorname{Eve}(g(\mathbf{r}))=1]\right| \\
& =\left|\operatorname{Pr}_{\mathbf{r} \leftarrow R^{\prime}}\left[h^{*}(f(\mathbf{r}))=0\right]-\operatorname{Pr}_{\mathbf{r} \leftarrow R^{\prime}}\left[h^{*}(g(\mathbf{r}))=0\right]\right| \\
& =\left|\left\{\underset{\mathbf{r r} \leftarrow R^{\prime}}{\operatorname{Pr}}\left[\mathbf{r} \in S_{00}\left(h^{*}\right)\right]+\underset{\mathbf{r} \leftarrow R^{\prime}}{\operatorname{Pr}^{\prime}}\left[\mathbf{r} \in S_{01}\left(h^{*}\right)\right]\right\}-\left\{\underset{\mathbf{r} \leftarrow R^{\prime}}{\operatorname{Pr}_{1}}\left[\mathbf{r} \in S_{00}\left(h^{*}\right)\right]+\underset{\mathbf{r} \leftarrow R^{\prime}}{\operatorname{Pr}}\left[\mathbf{r} \in S_{10}\left(h^{*}\right)\right]\right\}\right| \\
& =\left|\underset{\mathbf{r} \leftarrow R^{\prime}}{\operatorname{Pr}^{\prime}}\left[\mathbf{r} \in S_{01}\left(h^{*}\right)\right]-\operatorname{Pr}_{\mathbf{r} \leftarrow R^{\prime}}\left[\mathbf{r} \in S_{10}\left(h^{*}\right)\right]\right| \\
& \geq \delta .
\end{aligned}
$$

Therefore, the source $\mathcal{R}_{n}$ is $(t, \delta)$-expressive.

Remark 1. Note that if the universe $\mathcal{C}$ is a subset of $\{0,1\}^{\text {poly(n)}}$, then the universal hash function family in the proof of Theorem 3 can be made efficient (in $n$ ). Hence, the distinguisher Eve can be made efficient as well. Therefore, there exists an efficient distinguisher Eve such that $|\underset{\mathbf{r} \leftarrow R}{\operatorname{Pr}}[\operatorname{Eve}(f(\mathbf{r}))=1]-\underset{\mathbf{r} \leftarrow R}{\operatorname{Pr}}[\operatorname{Eve}(g(\mathbf{r}))=1]| \geq \delta$. Namely, $f(R)$ is " $\delta$-computationally distinguishable" from $g(R)$.

Combining Theorem 3 with Theorems 1 and 2, we get

\section{Corollary 1.}

(a) If a source $\mathcal{R}_{n}$ is $(1, \delta)$-separable, then no $\left(\mathcal{R}_{n}, \delta\right)$-secure bit extractor exists.

(b) If a source $\mathcal{R}_{n}$ is $(1, \delta)$-separable, then no $\left(\mathcal{R}_{n}, \delta\right)$-secure bit encryption scheme exists.

(c) If a source $\mathcal{R}_{n}$ is $(2, \delta)$-separable, then no $\left(\mathcal{R}_{n}, \delta\right)$-secure weak bit commitment exists.

(d) If a source $\mathcal{R}_{n}$ is $(\log T+1, \delta)$-separable, then no $\left(\mathcal{R}_{n}, \delta\right)$-secure bit $T$-secret sharing exists.

(e) Assume $1 /(8 \rho) \leq \varepsilon \leq 1 / 4$ and the source $\mathcal{R}_{n}$ is $\left(\log \left(\frac{\rho \varepsilon}{\delta}\right)+5, \delta\right)$-separable, for some $2 \varepsilon \leq \delta \leq 1$. Then no $\left(\mathcal{R}_{n}, \varepsilon\right)$-differentially private and $\left(U_{n}, \rho\right)$-accurate mechanism for the Hamming weight queries exists. In particular, plugging $\delta=2 \varepsilon$ and $\delta=\frac{1}{2}$, respectively, this holds if either (e.1) $\mathcal{R}_{n}$ is $(4+\log (\rho), 2 \varepsilon)$-separable; or $\left(\right.$ e.2) $\mathcal{R}_{n}$ is $\left(6+\log (\rho \varepsilon), \frac{1}{2}\right)$-separable.

The above results are illustrated by several typical sources in Section 5 .

\subsection{Separability and Weak Bit Extraction}

In this section, we define weak bit extraction and show that weak bit extraction is equivalent to NONseparability. Then we propose its implications to privacy.

Recall, Bosley and Dodis [BD07] initiated the study of the following general question: does privacy inherently require "extractable" source of randomness? A bit more formally, if a given primitive $P$ admits $\left(\mathcal{R}_{n}, \delta\right)$-secure implementation, does it mean that one can construct a (deterministic, single- or multi-) bit extractor from $\mathcal{R}_{n}$ ?

They also obtained very strong affirmative answers to this question for several traditional privacy primitives, including (only multi-bit) encryption and commitment (but not secret sharing, for example). Here we make the observation that our impossibility results give an incomparable (to [BD07]) set of affirmative answers to this question. On the positive, our results apply to a much wider set of primitives $P$ (e.g., secretsharing, as well as even single-bit encryption and commitment). On the negative, we can only argue a rather weak kind of single-bit extraction (as opposed to [BD07], who showed traditional, and possibly multi-bit extraction). Our weak notion of extraction is defined below.

Definition 9. We say that Ext: $\{0,1\}^{n} \rightarrow\{0,1, \perp\}$ is $\left(\mathcal{R}_{n}, \delta, \tau\right)$-secure weak bit extractor if

(a) for every distribution $R \in \mathcal{R}_{n},\left|\operatorname{Pr}_{\mathbf{r} \leftarrow R}[\operatorname{Ext}(\mathbf{r})=1]-\operatorname{Pr}_{\mathbf{r} \leftarrow R}[\operatorname{Ext}(\mathbf{r})=0]\right|<\delta$;

(b) $\operatorname{Pr}_{\mathbf{r} \leftarrow U_{n}}[\operatorname{Ext}(\mathbf{r}) \neq \perp] \geq \tau$. 
We briefly discuss this notion, before showing our results. First, we notice that setting $\tau=1$ recovers the notion of traditional bit-extractor given in Definition 2. And, even for general $\tau<1$, the odds of outputting 0 or 1 are roughly the same, for any distribution $R$ in the source. However, now the extractor is also allowed to output a failure symbol $\perp$, which means that each of the above two probabilities can occur with probabilities noticeably smaller than $1 / 2$. Hence, to make it interesting, we also add the requirement that Ext does not output $\perp$ all the time. This is governed by the second parameter $\tau$ requiring that $\operatorname{Pr}_{\mathbf{r} \leftarrow R}[\operatorname{Ext}(\mathbf{r}) \neq \perp] \geq \tau$. Ideally, we would like this to be true for any distribution $R$ in the source. Unfortunately, we will see shortly that such a desirable guarantee will not be achievable in our setting (see Remark 2). Thus, to salvage a meaningful and realizable notion, we will only require that this non-triviality guarantee at least holds for $R \equiv U_{n}$. Namely, while we do not rule out the possibility that some particular distributions $R$ might force Ext to fail the extraction with high probability, we still ensure that: (a) when the extraction succeeds, the extracted bit is unbiased for any $R$ in the source; (b) the extraction succeeds with noticeable probability at least when $R$ is ("close to") the uniform distribution $U_{n}$.

We now observe that the notion of weak bit-extraction is simply a different way to express (the negation of) our notion of separability!

Lemma 1. $\mathcal{R}_{n}$ has a $\left(\mathcal{R}_{n}, \delta, 2^{-t}\right)$-secure weak bit extractor if and only if $\mathcal{R}_{n}$ is not $(t, \delta)$-separable.

Proof. We only prove that non-separability implies weak bit extraction, as the converse is clear because all our steps will be "if and only if".

Since $\mathcal{R}_{n}$ is not $(t, \delta)$-separable, then there are two sets $G$ and $B$ such that $G \cap B=\emptyset,|G \cup B| \geq 2^{n-t}$ and for all $R \in \overline{\mathcal{R}_{n}}$, we have $\left|\operatorname{Pr}_{\mathbf{r} \leftarrow R}[\mathbf{r} \in G]-\operatorname{Pr}_{\mathbf{r} \leftarrow R}[\mathbf{r} \in B]\right|<\delta$. Define

$$
\operatorname{Ext}(\mathbf{r}) \stackrel{\text { def }}{=} \begin{cases}1, & \text { if } \mathbf{r} \in G \\ 0, & \text { if } \mathbf{r} \in B \\ \perp, & \text { otherwise. }\end{cases}
$$

This is well defined since $G \cap B=\emptyset$, and it satisfies properties (a) and (b) of weak bit extractor, since $\delta>\left|\operatorname{Pr}_{\mathbf{r} \leftarrow R}[\mathbf{r} \in G]-\operatorname{Pr}_{\mathbf{r} \leftarrow R}[\mathbf{r} \in B]\right|=|\underset{\mathbf{r} \leftarrow R}{\operatorname{Pr}}[\operatorname{Ext}(\mathbf{r})=1]-\underset{\mathbf{r} \leftarrow R}{\operatorname{Pr}}[\operatorname{Ext}(\mathbf{r})=0]|$, while $\underset{\mathbf{r} \leftarrow U_{n}}{\operatorname{Pr}}[\operatorname{Ext}(\mathbf{r}) \neq \perp]=$ $|G \cup B| / 2^{n} \geq 2^{n-t} / 2^{n}=2^{-t}$.

We can now combine Lemma 1 with the counter-positive of Corollary 1 to get the following result:

\section{Theorem 4.}

(a) If $\left(\mathcal{R}_{n}, \delta\right)$-secure bit encryption exists, then $\left(\mathcal{R}_{n}, \delta, \frac{1}{2}\right)$-secure weak bit-extraction exists.

(b) If $\left(\mathcal{R}_{n}, \delta\right)$-secure weak bit commitment exists, then $\left(\mathcal{R}_{n}, \delta, \frac{1}{4}\right)$-secure weak bit extraction exists.

(c) If $\left(\mathcal{R}_{n}, \delta\right)$-secure bit $T$-secret-sharing exists, then $\left(\mathcal{R}_{n}, \delta, \frac{1}{2 T}\right)$-secure weak bit extraction exists.

(d) If $\left(\mathcal{R}_{n}, \varepsilon\right)$-differentially private and $\left(U_{n}, \rho\right)$-accurate mechanism for the Hamming weight queries exists, then $\left(\mathcal{R}_{n}, 2 \varepsilon, \frac{1}{16 \rho}\right)$-secure weak bit extraction exists.

It is also instructive to see the explicit form of our weak bit extractor. For example, in the case of bit encryption (part (a), other examples similar), we get

$$
\operatorname{Ext}(\mathbf{r}) \stackrel{\text { def }}{=}\left\{\begin{array}{l}
1, \text { if } h^{*}\left(\operatorname{Enc}_{\mathbf{r}}(1)\right)=1 \text { and } h^{*}\left(\operatorname{Enc}_{\mathbf{r}}(0)\right)=0 \\
0, \text { if } h^{*}\left(\operatorname{Enc}_{\mathbf{r}}(1)\right)=0 \text { and } h^{*}\left(\operatorname{Enc}_{\mathbf{r}}(0)\right)=1 \\
\left.\perp, \text { otherwise (i.e., if } h^{*}\left(\operatorname{Enc}_{\mathbf{r}}(1)\right)=h^{*}\left(\operatorname{Enc}_{\mathbf{r}}(0)\right)\right)
\end{array}\right.
$$

where $h^{*}$ is the boolean universal hash function from the proof of Theorem 3, chosen as to ensure that

$$
\operatorname{Pr}_{\mathbf{r} \leftarrow U_{n}}[\operatorname{Ext}(\mathbf{r}) \neq \perp]=\operatorname{Pr}_{\mathbf{r} \leftarrow U_{n}}\left[h^{*}\left(\operatorname{Enc}_{\mathbf{r}}(0)\right) \neq h^{*}\left(\operatorname{Enc}_{\mathbf{r}}(1)\right)\right] \geq \frac{1}{2} .
$$

In particular, when the bit encryption (resp. commitment, secret sharing, DP mechanism) is computationally efficient (in $n$ ), our bit extractor is efficient as well. This means that even computationally secure analogs of encryption (commitment, secret sharing, DP mechanism) imply efficient, statistically secure weak bit extraction. 
Remark 2. As we mentioned, the major weakness of our weak bit extraction definition comes from the fact that the non-triviality condition $\underset{\mathbf{r} \leftarrow R}{\operatorname{Pr}}[\operatorname{Ext}(\mathbf{r}) \neq \perp] \geq \tau$ is only required for $R \equiv U_{n}$. Unfortunately, we observe that the analog of Theorem 4.(a)-(c) is no longer true if we require the extraction non-triviality to hold for all $R \in \mathcal{R}_{n}$. Indeed, this stronger notion of $\left(\mathcal{R}_{n}, \delta, \tau\right)$-secure weak bit extraction clearly implies traditional $\left(\mathcal{R}_{n}, 1+\delta-\tau\right)$-secure bit extraction (by mapping $\perp$ to 1$)$. On the other hand, Dodis and Spencer [DS02] gave an example of a source $\mathcal{R}_{n}$ for which, for any $\varepsilon>0$, there exists $\left(\mathcal{R}_{n}, \varepsilon\right.$ )-secure bit encryption (and hence, weak commitment and 2-secret sharing) scheme, but no $\left(\mathcal{R}_{n}, 1-2^{1-n / 2}\right)$-secure bit-extraction. Thus, the only analogs of Theorem 4.(a)-(c) we could hope to prove using the strengthened notion of weak bit extraction would have to satisfy $\tau \leq \delta+2^{1-n / 2}$, which is not a very interesting weak bit extraction scheme (e.g., if $\delta$ is "negligible", then the extraction succeeds with "negligible" probability as well). ${ }^{9}$

\section{$5 \quad$ Privacy with Several Typical Imperfect Sources}

Now we define several imperfect sources $\mathcal{R}_{n}$ : the $(k, n)$-source [CG88], $n$-bit $(k, m)$-block source [CG88], $(\gamma, n)$-Santha-Vazirani source [SV86], and $(\gamma, b, n)$-Bias-Control Limited (BCL) source [Dod01] as follows. Then we prove that several weak sources (i.e., Weak $(k, n), \mathcal{B l o c k}(k, m, n), \mathcal{S} \mathcal{V}(\gamma, n)$, and $\mathcal{B C} \mathcal{L}(\gamma, b, n))$ are separable. Based on this result, we show that the sources $\mathcal{W} e a k(\gamma, n), \mathcal{B} \operatorname{lock}(k, m, n), \mathcal{S} \mathcal{V}(\gamma, n)$, and $\mathcal{B C} \mathcal{L}(\gamma, b, n)$ are all expressive. Afterwards, we study the impossibility of traditional privacy and differential privacy with weak, block and BCL sources, and explain why the SV source does not work. Finally, we compare the impossibility of traditional privacy and differential privacy.

Definition 10. The $(k, n)$-source is defined by $\mathcal{W} e a k(k, n) \stackrel{\text { def }}{=}\left\{R \mid \mathbf{H}_{\infty}(R) \geq k\right.$, where $R$ is over $\left.\{0,1\}^{n}\right\}$.

Block sources are generalizations of weak sources, allowing $n / m$ blocks $R_{1}, \ldots, R_{n / m}$ each having $k$ fresh bits of entropy. ${ }^{10}$

Definition 11. Let $m$ divide $n$, and $R_{1}, R_{2}, \ldots, R_{n / m}$ be a sequence of Boolean random variables over $\{0,1\}^{m}$. A probability distribution $R=\left(R_{1}, R_{2}, \ldots, R_{n / m}\right)$ over $\{0,1\}^{n}$ is an $n$-bit $(k, m)$-Block distribution, denoted by $\operatorname{Block}(k, m, n)$, if for all $i \in[n / m]$ and for every $s_{1}, \ldots, s_{i-1} \in\{0,1\}^{m}$, we have

$$
\mathbf{H}_{\infty}\left(R_{i} \mid R_{1} \ldots R_{i-1}=s_{1} \ldots s_{i-1}\right) \geq k .
$$

We define the $n$-bit $(k, m)$-Block source Block $(k, m, n)$ to be the set of all $n$-bit $(k, m)$-Block distributions.

Hence, weak sources correspond to $m=n$ (i.e., one block). From the other extreme, Snatha-Vazirani sources correspond to 1-bit blocks (i.e., $m=1$ ). In this case, it is customary to express the imperfectness of the source as the function of its "bias" $\gamma$ instead of min-entropy $k$. Of course, for 1-bit random variables bias and min-entropy as related by $2^{-k}=(1+\gamma) / 2$. We get the following definition:

Definition 12. Let $r_{1}, r_{2}, \ldots, r_{n}$ be a sequence of Boolean random variables and $0 \leq \gamma<1$. A probability distribution $R=\left(r_{1}, r_{2}, \ldots, r_{n}\right)$ over $\{0,1\}^{n}$ is an $n$-bit $\gamma$-Santha-Vazirani (SV) distribution, denoted by $S V(\gamma, n)$, if for all $i \in[n]$ and for every string $s \in\{0,1\}^{i-1}$, we have

$$
\frac{1-\gamma}{2} \leq \operatorname{Pr}\left[r_{i}=1 \mid r_{1} r_{2} \ldots r_{i-1}=s\right] \leq \frac{1+\gamma}{2} .
$$

We define the $n$-bit $\gamma$-SV source $\mathcal{S} \mathcal{V}(\gamma, n)$ to be the set of all $n$-bit $\gamma$-SV distributions.

Finally, we define BCL sources [Dod01].

\footnotetext{
${ }^{9}$ For differential privacy (part (d)), we do not have an analog of the counter-example in [DS02], and anyway the value $\tau=O(1 / \rho) \ll \delta=O(\varepsilon)$ (so no contradiction). Of course, this does not imply that a stronger bit extraction result should be true; only that it is not definitely false.

${ }^{10}$ For consistency with prior work, we only assume that $R_{i}$ has $k$ fresh bits conditioned on the prior blocks, but our impossibility results easily extend to the case when we condition on both the past and the future blocks.
} 
Definition 13. Assume that $0 \leq \gamma<1$. The $(\gamma, b, n)$-Bias-Control Limited $(B C L)$ source $\mathcal{B C} \mathcal{L}(\gamma, b, n)$ generates $n$ bits $r_{1}, r_{2}, \ldots, r_{n}$, where for all $i \in[n]$, the value of $r_{i}$ can depend on $r_{1}, r_{2}, \ldots, r_{i-1}$ in one of the following two ways:

(a) $r_{i}$ is determined by $r_{1}, r_{2}, \ldots, r_{i-1}$, but this can happen for at most $b$ bits. This rule of determining a bit is called an intervention.

(b) $\frac{1-\gamma}{2} \leq \operatorname{Pr}\left[r_{i}=1 \mid r_{1} r_{2} \ldots r_{i-1}\right] \leq \frac{1+\gamma}{2}$.

Every distribution over $\{0,1\}^{n}$ generated from $\mathcal{B C} \mathcal{L}(\gamma, b, n)$ is called a $(\gamma, b, n)$-BCL distribution $B C L(\gamma, b, n)$.

In particular, when $b=0, \mathcal{B C} \mathcal{L}(\gamma, b, n)$ degenerates into $\mathcal{S} \mathcal{V}(\gamma, n)$ [SV86]; when $\gamma=0$, it yields the sequential-bit-fixing source of Lichtenstein, Linial, and Saks [LLS89].

\subsection{Separability Results}

In the following, we propose that the above sources are separable. It should be noted that: (a) The results for the weak and SV sources are implicitly known; (b) The BCL source was not considered before, but it is not hard to prove its separability given careful application of prior work; (c) The separability of the block source is new. It was not considered before because the SV source is a block source with each block of length 1, and [MP90,DOPS04] showed traditional privacy impossible even with the SV source (hence with the block source). But in light of [DLMV12], where differential privacy is possible with the SV source, we find it important to precisely figure out the separability of the block source. A naive approach would be to employ the $\gamma$-biased half-space source (see the appendix), introduced by [RVW04] and [DOPS04], which is both $\gamma$-SV and $\left(m-\log \frac{1+\gamma}{1-\gamma}, m\right)$-block sources. We can easily conclude that $(1) \mathcal{S V}(\gamma, n)$ is $\left(t, \frac{\gamma}{2^{t+1}}\right)$-separable, and (2) $\mathcal{B}$ lock $(k, m, n)$ is $\left(t, \frac{2^{m-k}-1}{2^{t+1} \cdot\left(2^{m-k}+1\right)}\right)$-separable. However, these results are somewhat sub-optimal. Instead, we introduce a new separability bound for block sources in Lemma 2 (b), and use it to get an improved result about the SV sources as well.

\section{Lemma 2.}

(a) Assume that $k \leq n-1$. Then $\mathcal{W} e a k(k, n)$ is $(t, 1)$-separable when $k \leq n-t-1$, and $\left(t, 2^{n-t-k-1}\right)-$ separable when $n-t-1<k \leq n-1$. In particular, it's $\left(t, \frac{1}{2}\right)$-separable when $k \leq n-t$.

(b) $\operatorname{Block}(k, m, n)$ is $\left(t, \frac{1}{1+2^{t+1} \cdot\left(\frac{2^{k}-1}{2^{m}-2^{k}}\right)}\right)$-separable. In particular, it is $\left(t, 1 /\left(1+2^{2+t+k-m}\right)\right)$-separable when $k \leq m-1$ (and, hence, $\left(t, \frac{1}{2}\right)$-separable when $k \leq m-t-2$ ).

(c) $\mathcal{S} \mathcal{V}(\gamma, n)$ is $\left(t, \frac{\gamma}{2^{t}}\right)$-separable.

(d) $\mathcal{B C L}(\gamma, b, n)$ is $\left(t, 1-\frac{2^{t+2}}{(1+\gamma)^{b}}\right)$-separable. In particular, it is $\left(t, \frac{1}{2}\right)$-separable for $b \geq \frac{t+3}{\log (1+\gamma)}=\Theta\left(\frac{t+1}{\gamma}\right)$.

Proof. Let $G, B \subseteq\{0,1\}^{n}$ where $G \cap B=\emptyset$ and $|G \cup B| \geq 2^{n-t}$. Without loss of generality, assume that $|G| \geq|B|$. Then $|\bar{G}| \geq 2^{n-t-1}$.

(a) Case 1: Assume that $k \leq n-t-1$. Pick any $S \subset\{0,1\}^{n}$ of size $|S|=2^{k}$ such that $S \subseteq G$. Then

$$
\operatorname{Pr}_{\mathbf{r} \leftarrow U_{S}}[\mathbf{r} \in G]-\operatorname{Pr}_{\mathbf{r} \leftarrow U_{S}}[\mathbf{r} \in B]=1-0=1 .
$$

Case 2: Assume that $n-t-1<k \leq n-1$.

Case 2.1: Suppose that $|G| \leq 2^{k}$. Then $|B|+2^{k} \leq|G|+2^{k} \leq 2^{k}+2^{k} \leq 2^{n}$. Choose a set $S \subset\{0,1\}^{n}$ of size $|S|=2^{k}$ such that $G \subseteq S$ and $B \cap S=\emptyset$. Then

$$
\underset{\mathbf{r} \leftarrow U_{S}}{\operatorname{Pr}}[\mathbf{r} \in G]-\operatorname{Pr}_{\mathbf{r} \leftarrow U_{S}}[\mathbf{r} \in B]=\frac{1}{2^{k}} \cdot|G|-0 \geq 2^{n-t-k-1} .
$$

Case 2.2: Now assume that $|G|>2^{k}$. Then pick any $S \subset\{0,1\}^{n}$ of size $|S|=2^{k}$ such that $S \subset G$. Then

$$
\operatorname{Pr}_{\mathbf{r} \leftarrow U_{S}}[\mathbf{r} \in G]-\operatorname{Pr}_{\mathbf{r} \leftarrow U_{S}}[\mathbf{r} \in B]=1-0=1
$$


Assume that $k \leq n-t$. If $k \leq n-t-1$, it can be reduced to Case 1 . Otherwise, it can be reduced to Case 2.

(b) Define $R$ as follows. $\operatorname{Pr}[R=r]=q$ when $r \notin G$, and $\operatorname{Pr}[R=r]=q\left(2^{m}-1\right) /\left(2^{k}-1\right)$ when $r \in G$, where $q$ is chosen such that $\left(2^{n}-|G|\right) \cdot q+|G| \cdot q\left(2^{m}-1\right) /\left(2^{k}-1\right)=1$, which is equivalent to

$$
q\left(|G| \cdot \frac{2^{m}-2^{k}}{2^{k}-1}+2^{n}\right)=1
$$

First, we claim that $R$ is a valid $n$-bit $(k, m)$-Block-Source. For this, we show a slightly stronger statement (see Footnote 10): for every $i \in[n / m]$ and $s_{1}, \ldots, s_{i-1}, s_{i+1}, \ldots, s_{n / m} \in\{0,1\}^{m}$, we have

$$
\mathbf{H}_{\infty}\left(R_{i} \mid R_{1} \ldots R_{i-1} R_{i+1} \ldots R_{n / m}=s_{1} \ldots s_{i-1} s_{i+1} \ldots s_{n / m}\right) \geq k
$$

(Correspondingly, $\mathbf{H}_{\infty}\left(R_{i} \mid R_{1} \ldots R_{i-1}=s_{1} \ldots s_{i-1}\right) \geq k$.)

Indeed, for every $s_{i} \in\{0,1\}^{m}$,

$$
\begin{aligned}
& \operatorname{Pr}\left[R_{i}=s_{i} \mid R_{1} \ldots R_{i-1} R_{i+1} \ldots R_{n / m}=s_{1} \ldots s_{i-1} s_{i+1} \ldots s_{n / m}\right] \\
= & \frac{\operatorname{Pr}\left[R_{1} \ldots R_{i-1} R_{i} R_{i+1} \ldots R_{n / m}=s_{1} \ldots s_{i-1} s_{i} s_{i+1} \ldots s_{n / m}\right]}{\sum_{s_{i}^{\prime} \in\{0,1\}^{m}} \operatorname{Pr}\left[R_{1} \ldots R_{i-1} R_{i} R_{i+1} \ldots R_{n / m}=s_{1} \ldots s_{i-1} s_{i}^{\prime} s_{i+1} \ldots s_{n / m}\right]} \\
\leq & \frac{q\left(2^{m}-1\right) /\left(2^{k}-1\right)}{q\left(2^{m}-1\right) /\left(2^{k}-1\right)+q\left(2^{m}-1\right)}=2^{-k}
\end{aligned}
$$

as claimed.

Next, since $|G| \geq|B|$, we have

$$
\left|\operatorname{Pr}_{\mathbf{r} \leftarrow R}[\mathbf{r} \in G]-\operatorname{Pr}_{\mathbf{r} \leftarrow R}[\mathbf{r} \in B]\right|=|G| \cdot \frac{q\left(2^{m}-1\right)}{2^{k}-1}-|B| \cdot q \geq q \cdot|G| \cdot \frac{2^{m}-2^{k}}{2^{k}-1} .
$$

Comparing to Equation (2) and letting $\alpha=q \cdot|G| \cdot \frac{2^{m}-2^{k}}{2^{k}-1}, \beta=q \cdot 2^{n}$, we need to lower bound $\alpha$ subject to the constraints: (1) $\alpha+\beta=1$; (2) $\beta=\alpha \cdot \frac{\left(2^{k}-1\right) 2^{n}}{\left(2^{m}-2^{k}\right)|G|} \leq \alpha \cdot \frac{2^{t+1}\left(2^{k}-1\right)}{2^{m}-2^{k}}$, where we used $|G| \geq 2^{n-t-1}$. Combining (1) and (2), we get

$$
1=\alpha+\beta \leq \alpha\left(1+2^{t+1} \cdot \frac{2^{k}-1}{2^{m}-2^{k}}\right)
$$

which solves precisely to the lower bound on $\alpha$ claimed in part (b).

When $k \leq m-1$, we get $\left(2^{k}-1\right) /\left(2^{m}-2^{k}\right) \leq 2^{k} / 2^{m-1}=2^{k-m+1}$, which gives the claimed bound

$$
\frac{1}{1+2^{t+1} \cdot\left(\frac{2^{k}-1}{2^{m}-2^{k}}\right)} \geq \frac{1}{1+2^{2+t+k-m}} .
$$

(c) We simply use the bound from part (b) for the special case when $m=1$ and $2^{-k}=(1+\gamma) / 2$. Then $2^{k}-1=(1-\gamma) /(1+\gamma), 2^{m}-2^{k}=2-2 /(1+\gamma)=2 \gamma /(1+\gamma)$, and we get $(t, \delta)$-separability with

$$
\delta=\frac{1}{1+2^{t+1} \cdot \frac{1-\gamma}{2 \gamma}}=\frac{\gamma}{2^{t}-\gamma\left(2^{t}-1\right)} \geq \frac{\gamma}{2^{t}}, \text { since } t \geq 0
$$

(d) We start by recalling the following auxiliary result from [Dod01].

Given a Boolean function $f_{e}:\{0,1\}^{n} \rightarrow\{0,1\}$, it is associated with an event $E$ such that " $E$ happens $\Longleftrightarrow f_{e}(\mathbf{x})=1 "$, where $\mathbf{x} \in\{0,1\}^{n}$. The natural probability $p$ of $E$ is defined as the probability that $E$ happens for an ideal source (in our case, emitting $n$ perfect unbiased bits). More formally,

$$
p=\operatorname{Pr}_{\mathbf{r} \leftarrow U_{n}}\left[f_{e}(\mathbf{r})=1\right]=\operatorname{Pr}_{\mathbf{r} \leftarrow U_{n}}[\text { E happens }]
$$


We then say that $E$ (or $f_{e}$ ) is $p$-sparse. We define the set of all $p$-sparse events (or Boolean functions) as $\mathcal{E}$.

We view the source $\mathcal{B C} \mathcal{L}(\gamma, b, n)$ as an adversary $\mathcal{A}$ who can influence the ideal behavior of the source by applying rules (A) and (B) of Definition 13. Our goal is to see whether our adversary $\mathcal{A}$ has enough power to significantly influence the occurrence of the event $E$. For a given number of interventions $b$, to obtain the largest probability of "success" that $\mathcal{A}$ can achieve (i.e., the largest probability that any $p$-sparse event $E$ happens for $\mathcal{B C L}(\gamma, b, n))$, we first study the complement notion of "smallest probability of failure" and get the following claim.

Claim. ([Dod01]) Let $F(p, n, b) \stackrel{\text { def }}{=} \max _{e \in \mathcal{E}} \min _{R \in \mathcal{B C} \mathcal{L}(\gamma, b, n)} \operatorname{Pr}_{\mathbf{r} \leftarrow R}\left[f_{e}(\mathbf{r})=0\right]$. Then $F(p, n, b) \leq \frac{1}{p \cdot(1+\gamma)^{b}}=2^{\log \frac{1}{p}-\Theta(\gamma b)}$.

In other words, if $b$ is "high enough" (i.e., $b \gg \frac{1}{\gamma} \log \frac{1}{p}$ ), then the imperfect source attacker $\mathcal{A}$ can force any $p$-sparse event to happen with probability very close to 1 .

Now let's come back to our lemma. Define the function $f_{e}:\{0,1\}^{n} \rightarrow\{0,1\}$ as follows.

$$
f_{e}(\mathbf{r})= \begin{cases}1, & \text { if } \mathbf{r} \in G \\ 0, & \text { otherwise }\end{cases}
$$

Then from the above claim, we have $\min _{R \in \mathcal{B C} \mathcal{L}(\gamma, b, n)} \underset{\mathbf{r} \leftarrow R}{\operatorname{Pr}}\left[f_{e}(\mathbf{r})=0\right] \leq \frac{1}{\frac{|G|}{2^{n}} \cdot(1+\gamma)^{b}}$.

Thus, there exists a $(\gamma, b, n)-$ BCL distribution $R_{0}$ such that

$$
\operatorname{Pr}_{\mathbf{r} \leftarrow R_{0}}\left[f_{e}(\mathbf{r})=0\right]=\min _{R \in \mathcal{B C} \mathcal{L}(\gamma, b, n)} \operatorname{Pr}_{\mathbf{r} \leftarrow R}\left[f_{e}(\mathbf{r})=0\right] \leq \frac{1}{\frac{|G|}{2^{n}} \cdot(1+\gamma)^{b}} .
$$

Hence,

$$
\begin{gathered}
\operatorname{Pr}_{\mathbf{r} \leftarrow R_{0}}[\mathbf{r} \in G]=\operatorname{Pr}_{\mathbf{r} \leftarrow R_{0}}\left[f_{e}(\mathbf{r})=1\right] \geq 1-\frac{1}{\frac{|G|}{2^{n}} \cdot(1+\gamma)^{b}} . \\
\quad \operatorname{Pr}_{\mathbf{r} \leftarrow R_{0}}[\mathbf{r} \in B] \leq \operatorname{Pr}_{\mathbf{r} \leftarrow R_{0}}\left[f_{e}(\mathbf{r})=0\right] \leq \frac{1}{\frac{|G|}{2^{n}} \cdot(1+\gamma)^{b}} .
\end{gathered}
$$

Correspondingly, $\operatorname{Pr}_{\mathbf{r} \leftarrow R_{0}}[\mathbf{r} \in G]-\operatorname{Pr}_{\mathbf{r} \leftarrow R_{0}}[\mathbf{r} \in B] \geq 1-\frac{2}{\frac{\left.T^{\prime}\right]}{2^{n}} \cdot(1+\gamma)^{b}} \geq 1-\frac{2^{t+2}}{(1+\gamma)^{b}}$. Therefore, $\mathcal{B C L}(\gamma, b, n)$ is $\left(t, 1-\frac{2^{t+2}}{(1+\gamma)^{b}}\right)$-separable.

Let $\frac{2^{t+2}}{(1+\gamma)^{b}} \leq \frac{1}{2}$, that is, $b \geq \frac{t+3}{\log (1+\gamma)}$. Therefore, $\mathcal{B C L}(\gamma, b, n)$ is $\left(t, \frac{1}{2}\right)$-separable if $b \geq \frac{t+3}{\log (1+\gamma)}$.

\subsection{Implications to Traditional and Differential Privacy}

Impossibility of Traditional Privacy. From Lemma 2 and Corollary 1 (a)-(d), we conclude:

Theorem 5. For the following values of $\delta$, shown in Table 1 , no $\left(\mathcal{R}_{n}, \delta\right)$-secure cryptographic primitive $P$ exists, where $\mathcal{R}_{n} \in\{\mathcal{W}$ eak $(k, n), \mathcal{B l o c k}(m-1, m, n), \mathcal{S} \mathcal{V}(\gamma, n), \mathcal{B C} \mathcal{L}(\gamma, b, n)\}$ and $P \in\{$ bit extractor, bit encryption scheme, weak bit commitment, bit $T$-secret sharing $\}$.

We notice that, while the impossibility results for the BCL source are new, the prior work of [MP90,DOPS04] already obtained similar results for the weak, block and SV sources. However, our results still offer some improvements over the works of [MP90,DOPS04]. First, unlike the work of [MP90], our distinguisher is efficient (see Remark 1), ruling out even computationally secure encryption, commitment, and secret sharing schemes. Second, unlike the work of [DOPS04], our lower bound on $\delta$ does not depend on the sizes of ciphertext/commitment/shares. In particular, while [DOPS04] used a bit-by-bit hybrid argument to show their impossibility results, our proof of Theorem 3 used a more clever "universal hashing trick". More importantly, instead of focusing the entire proof on some specific weak/block/SV sources [MP90,DOPS04], our impossibility results for such sources were obtained in a more modular manner, making these proofs somewhat more illuminating. 


\begin{tabular}{|l|c|c|c|c|}
\hline \multicolumn{1}{|c|}{$P$} & bit extractor & bit encryption scheme & weak bit commitment & bit $T$-secret sharing \\
\hline $\mathcal{W}$ eak $(k, n)$ & 1, if $k \leq n-2$ & 1, if $k \leq n-2$ & 1, if $k \leq n-3$ & 1, if $k \leq n-\log T-2$ \\
\hline $\mathcal{W}$ eak $(n-1, n)$ & $\frac{1}{2}$ & $\frac{1}{2}$ & $\frac{1}{4}$ & $\frac{1}{2 T}$ \\
\hline $\mathcal{B} \operatorname{lock}(m-1, m, n)$ & $\frac{1}{5}$ & $\frac{1}{5}$ & $\frac{1}{9}$ & $\frac{1}{4 T+1}$ \\
\hline $\mathcal{S} \mathcal{V}(\gamma, n)$ & $\frac{\gamma}{2}$ & $\frac{\gamma}{2}$ & $\frac{\gamma}{4}$ & $\frac{\gamma}{2 T}$ \\
\hline $\mathcal{B C} \mathcal{L}(\gamma, b, n)$ & $\frac{1}{2}$, if $b \geq \frac{4}{\log (1+\gamma)}$ & $\frac{1}{2}$, if $b \geq \frac{4}{\log (1+\gamma)}$ & $\frac{1}{2}$, if $b \geq \frac{5}{\log (1+\gamma)}$ & $\frac{1}{2}$, if $b \geq \frac{\log T+4}{\log (1+\gamma)}$ \\
\hline
\end{tabular}

Table 1. Values of $\delta$ for which no $\left(\mathcal{R}_{n}, \delta\right)$-secure cryptographic primitive $P$ exists.

Impossibility of Differential Privacy with the Weak, Block and BCL sources. Now we apply the impossibility results of differential privacy to the sources $\mathcal{W e a k}(k, n), \mathcal{B l o c k}(k, m, n)$, and $\mathcal{B C} \mathcal{L}(\gamma, b, n)$. In particular, by combining Corollary 1 (e.2) with Lemma 2 (a), Lemma 2 (b), and Lemma 2 (d), respectively, we get

Theorem 6. For the following sources $\mathcal{R}_{n}$, no $\left(\mathcal{R}_{n}, \varepsilon\right)$-differentially private and $\left(U_{n}, \rho\right)$-accurate mechanisms for the Hamming weight queries exist:

(a) Weak $(k, n)$ where $k \leq n-\log (\varepsilon \rho)-6$;

(b) $\operatorname{Block}(k, m, n)$ where $k \leq m-\log (\varepsilon \rho)-8$;

(c) $\mathcal{B C L}(\gamma, b, n)$ where $b \geq \frac{\log (\varepsilon \rho)+9}{\log (1+\gamma)}=\Omega\left(\frac{\log (\varepsilon \rho)+1}{\gamma}\right)$.

We discuss the (non-)implications to the SV source below, but notice the strength of these negative results the moment the source becomes a little bit more "adversarial" as compared to the SV source. In particular, useful mechanisms in differential privacy (called "non-trivial" by [DLMV12]) aim to achieve utility $\rho$ (with respect to the uniform distribution) which only depends on the differential privacy $\varepsilon$, and not on the size $N$ of the database $D$. This means that the value $\log (\varepsilon \rho)$ is typically upper bounded by some constant $c=O(1)$. For such "non-trivial" mechanisms, our negative results say that differential privacy is impossible with (1) weak sources even when the min-entropy $k=n-O(1)$; (2) block sources even when the min-entropy $k=m-O(1)$; (3) BCL sources even when the number of interventions $b=\Omega(1)$. So what prevented us from strong impossibility for the SV sources, as is expected given the feasibility results of [DLMV12]? The short answer is that the separability of the SV sources given by Lemma 2 (c) is just not good enough to yield very strong results, as we explain now.

(Non-)Implications FOR The SV source. We observe that Theorem 2 can't be applied to the SV source, as $\mathcal{S} \mathcal{V}(\gamma, n)$ is only $(t, \delta)$-expressive for $\delta=\frac{\gamma}{2^{t+1}}$, which means that $2^{t} \delta=O(\gamma)$. In contrast, to apply Theorem 2 we need $2^{t} \delta \geq \Omega(\rho \varepsilon)$. Thus, to have any hope, we need, $\rho=O(\gamma / \varepsilon)$, but this violates our pre-condition (used in the proof) that $\rho \geq 1 /(8 \varepsilon)$. In fact, a simple reworking of the proof of Theorem 2 (omitted) can be used to show that if there exists a $(\mathcal{S V}(\gamma, n), \varepsilon)$-differentially private and $\left(U_{n}, \rho\right)$-accurate mechanism for the Hamming weight queries, then $\rho>\frac{\gamma}{32 \cdot \varepsilon}=\Omega\left(\frac{\gamma}{\varepsilon}\right)$.

Unfortunately, this implication that we get is quite weak, because we can get a stronger result, even if $\mathcal{R}_{n}$ consists only of the uniform distribution $U_{n}$. We present this well known folklore result for completeness.

Lemma 3. Assume that the mechanism $M$ is $\left(U_{n}, \varepsilon\right)$-differentially private and $\left(U_{n}, \rho\right)$-accurate for the Hammimg weight queries. Then $\rho \geq \frac{1}{e+1} \cdot \frac{1}{\varepsilon}=\Omega\left(\frac{1}{\varepsilon}\right)$.

Proof. For any $D, D^{\prime} \in \mathcal{D}$, let $\beta \stackrel{\text { def }}{=} \frac{\mathbb{E}_{\mathbf{r}} \leftarrow R}{\mathbb{E}_{\mathbf{r} \leftarrow R}\left[M\left(D^{\prime}, \mathbf{r}\right)\right]}$. From Definition 7 , we have $\beta \geq \frac{w t\left(D^{\prime}\right)-\rho}{w t(D)+\rho}$. By Definition 6 , we obtain

$$
\beta=\frac{\sum_{z} z \operatorname{Pr}_{\mathbf{r} \leftarrow R}\left[M\left(D^{\prime}, \mathbf{r}\right)=z\right]}{\sum_{z} z \operatorname{Pr}_{\mathbf{r} \leftarrow R}[M(D, \mathbf{r})=z]} \leq \frac{\sum_{z} z e^{\varepsilon \cdot \Delta\left(D, D^{\prime}\right)} \operatorname{Pr}_{\mathbf{r} \leftarrow R}[M(D, \mathbf{r})=z]}{\sum_{z} z \operatorname{Pr}_{\mathbf{r} \leftarrow R}[M(D, \mathbf{r})=z]}=e^{\varepsilon \cdot \Delta\left(D, D^{\prime}\right)} .
$$


Therefore,

$$
\frac{w t\left(D^{\prime}\right)-\rho}{w t(D)+\rho} \leq e^{\varepsilon \cdot \Delta\left(D, D^{\prime}\right)} .
$$

Take a specific $D$ such that $w t(D)=0$. Let $\operatorname{Ball}(D, \alpha)=\left\{D^{\prime}: \Delta\left(D, D^{\prime}\right) \leq \alpha\right\}$. Then

$$
\forall D^{\prime} \in \operatorname{Ball}\left(D, \frac{1}{\varepsilon}\right) \Rightarrow \frac{w t\left(D^{\prime}\right)-\rho}{\rho} \leq e^{\varepsilon \cdot \Delta\left(D, D^{\prime}\right)} \leq e \Rightarrow \rho \geq \frac{1}{e+1} w t\left(D^{\prime}\right) .
$$

Taking $D^{\prime}$ such that $w t\left(D^{\prime}\right)=\frac{1}{\varepsilon}$, we get $\rho \geq \frac{1}{e+1} \cdot \frac{1}{\varepsilon}$.

Thus, our technique cannot yield any results for the $\gamma$-SV source, which we didn't already know even for the uniform distribution. Of course, this is not surprising, because Dodis et al. [DLMV12] have shown that we can get $(\mathcal{S} \mathcal{V}(\gamma, n), \varepsilon)$-differentially private and $(\mathcal{S V}(\gamma, n), \rho)$-accurate mechanism for all counting queries (including the Hamming weight queries), where $\rho=\operatorname{poly}_{1 /(1-\gamma)}\left(\frac{1}{\varepsilon}\right) \gg \frac{1}{\varepsilon}$ and $\operatorname{pol}_{1 /(1-\gamma)}(x)$ denotes a polynomial whose degree and coefficients are fixed (and rather large) functions of $1 /(1-\gamma)$.

\subsection{Comparing Impossibility Results for Traditional and Differential Privacy}

In this section, we compare the impossibility of traditional privacy and differential privacy (see Table 2). For traditional privacy, we consider bit extractor, bit encryption scheme, weak bit commitment, and bit 2-secret

\begin{tabular}{|c|c|c|}
\hline Source & Traditional Privacy $\delta$ & Differential Privacy $\varepsilon \&$ Utility $\rho$ \\
\hline $\mathcal{B l o c k}(k, m, n)$ & Impossible if $\delta \leq \frac{1}{9}$, even if $k=m-1$ & Impossible if $k \leq m-\log (\varepsilon \rho)-O(1)$ \\
\hline $\mathcal{S V}(\gamma, n)$ & Impossible if $\delta=O(\gamma)$ & $\begin{array}{l}\text { Impossible if } \rho=O\left(\frac{1}{\varepsilon}\right) \text {, even for } U_{n} \\
\left.\text { (Possible if } \rho=\operatorname{poly}_{1 /(1-\gamma)}\left(\frac{1}{\varepsilon}\right) \gg \frac{1}{\varepsilon}\right)\end{array}$ \\
\hline $\mathcal{B C} \mathcal{L}(\gamma, b, n)$ & $\begin{array}{l}\text { Impossible if } \delta=O(\gamma) \text {, even if } b=0 \\
\text { Impossible if } \delta \leq \frac{1}{2} \text { and } b=\Omega\left(\frac{1}{\gamma}\right)\end{array}$ & Impossible if $b=\Omega\left(\frac{\log (\varepsilon \rho)+1}{\gamma}\right)$ \\
\hline
\end{tabular}
sharing (e.g., set $T=2$ for concreteness).

Table 2. Comparison about the Impossibility of Traditional Privacy and Differential Privacy.

In particular, while a very "structured" (and, hence, rather unrealistic) SV source is sufficient to guarantee loose, but non-trivial differential privacy, without guaranteeing (strong-enough) traditional privacy, once the source becomes more realistic (e.g., number of interventions $b$ becomes super-constant, or one removes the conditional entropy guarantee within different blocks), both notions of privacy become impossible extremely quickly. In other words, despite the surprising feasibility result of [DLMV12] regarding differential privacy with SV sources, the prevalent opinion that "privacy is impossible with realistic weak randomness" appears to be rather accurate.

Acknowledgments. The authors would like to thank Benjamin Fuller, Sasha Golovnev, Hamidreza Jahanjou, Zhoujun Li, Umut Orhan, and Abhishek Samanta.

Yevgeniy Dodis was partially supported by gifts from VMware Labs and Google, and NSF grants 1319051, 1314568, 1065288, and 1017471. Yanqing Yao was supported by the Scholarship Award for Excellent Doctoral Student granted by Ministry of Education 400618, and CSC grant 201206020063. 


\section{References}

$\left[\mathrm{ACM}^{+} 14\right]$ Per Austrin, Kai-Min Chung, Mohammad Mahmoody, Rafael Pass, and Karn Seth. On the Impossibility of Cryptography with Tamperable Randomness. CRYPTO, volume 8616 of LNCS, pages 462-479. Springer, 2014.

[ACRT99] Alexander E. Andreev, Andrea E.F. Clementi, José D.P. Rolim, and Luca Trevisan. Weak random sources, hitting sets, and BPP simulations. SIAM J. Comput., 28(6): 2103-2116, 1999.

[Blu86] Manuel Blum. Independent unbiased coin-flips from a correclated biased source-a finite state Markov chain. Combinatorica, 6(2): 97-108, 1986.

[BD07] Carl Bosley and Yevgeniy Dodis. Does privacy require true randomness? In Salil P. Vadhan, editor, TCC, volume 4392 of LNCS, pages 1-20. Springer, 2007.

$\left[\mathrm{BDK}^{+} 05\right]$ Xavier Boyen, Yevgeniy Dodis, Jonathan Katz, Rafail Ostrovsky, and Adam Smith. Secure remote authentication using biometric data. In Ronald Cramer, editor, Advances in Cryptology EUROCRYPT 2005, volume 3494 of LNCS, pages 147-163. Springer-Verlag, 2005.

[BH05] Boaz Barak and Shai Halevi. A model and architecture for pseudo-random generation with applications to /dev/random. In Proceedings of the 12th ACM Conference on Computer and Communication Security, pages 203-212, 2005.

[BST03] Boaz Barak, Ronen Shaltiel, and Eran Tromer. True random number generators secure in a changing environment. In Proceedings of the 5th Cryptographic Hardware and Embedded Systems, pages 166-180, 2003.

$\left[\mathrm{CFG}^{+} 85\right]$ Benny Chor, Joel Friedman, Oded Goldreich, Johan Håstad, Steven Rudich, and Roman Smolensky. The Bit Extraction Problem or $t$-resilient Functions. In Proc. of 26th FOCS, pages 396-407, 1985.

[CG88] Benny Chor and Oded Goldreich. Unbiased bits from sources of weak randomness and probabilistic communication complexity. SIAM J. Comput., 17(2): 230-261, 1988.

[CW79] J. Lawrence Carter and Mark N. Wegman. Universal Classes of Hash Functions. J. Comput. Syst. Sci., 18(2): 143-154, 1979.

[Dod01] Yevgeniy Dodis. New Imperfect Random Source with Applications to Coin-Flipping. ICALP 2001, pages 297-309.

[DKRS06] Yevgeniy Dodis, Jonathan Katz, Leonid Reyzin, and Adam Smith. Robust fuzzy extractors and authenticated key agreement from close secrets. In Cynthia Dwork, editor, CRYPTO, volume 4117 of LNCS, pages 232-250. Springer, 2006.

[DLMV12] Yevgeniy Dodis, Adriana López-Alt, Ilya Mironov, and Salil P. Vadhan. Differential Privacy with Imperfect Randomness. CRYPTO 2012, pages 497-516.

[DMNS06] Cynthia Dwork, Frank McSherry, Kobbi Nissim, and Adam Smith. Calibrating noise to sensitivity in private data analysis. In Shai Halevi and Tal Rabin, editors, TCC, volume 3876 of LNCS, pages 265-284. Springer, 2006.

[DOPS04] Yevgeniy Dodis, Shien Jin Ong, Manoj Prabhakaran, and Amit Sahai. On the (im)possibility of cryptography with imperfect randomness. FOCS 2004, pages 196-205.

[DORS08] Yevgeniy Dodis, Rafail Ostrovsky, Leonid Reyzin, and Adam Smith. Fuzzy extractors: How to generate strong keys from biometrics and other noisy data. SIAM Journal on Computing, 38(1): 97-139, 2008.

[DS02] Yevgeniy Dodis and Joel Spencer. On the (non)Universality of the One-Time Pad. FOCS 2002, pages 376-385.

[GKR04] Rosario Gennaro, Hugo Krawczyk, and Tal Rabin. Secure hashed diffie-hellman over non-ddh groups. In Christian Cachin and Jan Camenisch, editors, Advances in Cryptology EUROCRYPT 2004, volume 3027 of LNCS, pages 361-381. Springer-Verlag, 2004.

[Kra10] Hugo Krawczyk. Cryptographic Extraction and Key Derivation: The HKDF Scheme. In Tal Rabin, editor, Advances in Cryptology-CRYPTO 2010, volume 6223 of LNCS, pages 631-648. Springer-Verlag, 2010.

[LLS89] David Lichtenstein, Nathan Linial, and Michael E. Saks. Some extremal problems arising form discrete control processes. Combinatorica, 9(3): 269-287, 1989.

[MP90] James L. McInnes and Benny Pinkas. On the impossibility of private key cryptography with weakly random keys. In Alfred Menezes and Scott A. Vanstone, editors, CRYPTO, volume 537 of LNCS, pages 421-435. Springer, 1990. 
[MW97] Ueli M. Maurer and Stefan Wolf. Privacy amplification secure against active adversaries. In Burton S. Kaliski, Jr., editor, CRYPTO, volume 1294 of LNCS, pages 307-321. Springer, 1997.

[RVW04] Omer Reingold, Salil Vadhan, and Avi Widgerson. No Deterministic Extraction from SanthaVazirani Sources: a Simple Proof. http://windowsontheory.org/2012/02/21/nodeterministic-extractionfrom-santha-vazirani-sources-a-simple-proof/, 2004.

[SV86] Miklos Santha and Umesh V. Vazirani. Generating quasi-random sequences from semirandom sources. J. Comput. Syst. Sci., 33(1): 75-87, 1986.

[vN51] John von Neumann. Various techniques used in connection with random digits. In National Bureau of Standards, Applied Math. Series, 12: 36-38, 1951.

[VV85] Umesh V. Vazirani and Vijay V. Vazirani. Random polynomial time is equal to slightly random polynomial time. FOCS, pages 417-428, 1985.

[Zuc96] David Zuckerman. Simulating BPP using a general weak random source. Algorithmica, 16(4/5): 367-391, 1996.

\section{Appendix:}

Definition 14. Given $S \subset\{0,1\}^{n}$ of size $|S|=2^{n-1}$, and $0 \leq \gamma<1$, the distribution $R \stackrel{\text { def }}{=} H_{S}(\gamma, n)$ over $\{0,1\}^{n}$ is defined as

$$
\begin{cases}\operatorname{Pr}[R=\mathbf{r}]=(1+\gamma) \cdot 2^{-n}, & \text { if } \mathbf{r} \in S \\ \operatorname{Pr}[R=\mathbf{r}]=(1-\gamma) \cdot 2^{-n}, & \text { otherwise }\end{cases}
$$

The $\gamma$-biased half-space source $\mathcal{H}(\gamma, n)$ is defined as

$$
\mathcal{H}(\gamma, n) \stackrel{\text { def }}{=}\left\{H_{S}(\gamma, n) \mid S \subseteq\{0,1\}^{n} \text { and }|S|=2^{n-1}\right\}
$$

